\title{
Upper Silurian and Lower Devonian conodonts from Tafilalt, southeastern Morocco
}

\author{
MARIA G. CORRIGA, CARLO CORRADINI \& OTTO H. WALLISER ${ }^{\dagger}$
}

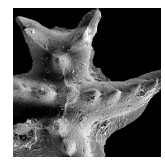

\begin{abstract}
The conodont association from three Silurian-Lower Devonian sections (Bou Tchrafine N2, Atrous 3 and Atrous 7) in the Tafilalt (southeast Morocco) are presented. The sections are constituted by cephalopod rich limestones ("Orthoceras limestones") and by crinoidal limestones ("Scyphocrinites limestones") cropping out within thick shaley sequences. The association includes 29 taxa belonging to 13 genera (Ancyrodelloides, Belodella, Dvorakia, Icriodus, Kockelella, Lanea, Oulodus, Ozarkodina, Pelekysgnathus, Polygnathoides, Pseudooneotodus, Wurmiella, Zieglerodina). The conodont fauna allows the recognition of seven conodont zones: ploeckensis and siluricus in the Ludlow, eosteinhornensis s.l., Lower detortus and Upper detortus in the Př́dolí, and hesperius and transitans in the Lochkovian. The age of the "Orthoceras limestones" is confirmed as middle Ludfordian (ploeckensis-siluricus zones), whereas it is stated that the "Scyphocrinites limestones" spans the Silurian/Devonian boundary. Some elements of the apparatus of Zieglerodina planilingua and the P2 element of Lanea omus are described. The phylogenetic relationships between genera Lanea and Ancyrodelloides are discussed. - Key words: conodonts, biostratigraphy, taxonomy, Silurian, Lower Devonian, Morocco.
\end{abstract}

Corriga, M.G., Corradini, C. \& WAlliser, O.H. 2014. Upper Silurian and Lower Devonian conodonts from Tafilalt, southeastern Morocco. Bulletin of Geosciences 89(1), 183-200 (7 figures, 3 tables). Czech Geological Survey, Prague. ISSN 1214-1119. Manuscript received July 29, 2013; accepted in revised form October 5, 2013; published online January 21, 2014; issued January 21, 2014.

Maria G. Corriga \& Carlo Corradini (corresponding author), Dipartimento di Scienze Chimiche e Geologiche, Universitàdi Cagliari,via Trentino 51, I-09129 Cagliari, Italy; maria.corriga@unica.it, corradin@unica.it•Otto H. Walliser, Geowissenschaftliches Zentrum der Universität, Abt. Geobiologie, Goldschmidt-Str. 3, D-37077 Göttingen, Germany

In the Tafilalt region, southeastern Morocco, rocks from Ordovician to Carboniferous are widely exposed. Among this sequence the Silurian and lowermost Devonian terms are the less investigated, being represented mainly by pelitic and marly sediments cropping out at the base of ridges constituted by Devonian limestones and sandstones (Hollard 1977). Carbonatic beds and lenses occur within the shales: in first approximation they are represented by cephalopod rich limestones ("Orthoceras limestones") and by crinoidal limestones ("Scyphocrinites limestones"). These beds are well known mainly for the perfectly preserved fossils, and for mining activities with commercial background that increased since the late 1970s.

Only a few stratigraphic data are available from these limestones: in first approximation the Orthoceras limestones are reported from the Ludlow to the Lochkovian, whereas the Scyphocrinites limestones occur across the $\mathrm{Si}$ lurian/Devonian boundary, but their precise age is still disputed on the basis of scarce data. Hollard (1977) placed the Silurian/Devonian boundary in the upper part of the Scyphocrinites limestones on the basis of the occurrence of
Monograptus uniformis in the Taouz section, in southern Tafilalt. This datum is confirmed by Haude \& Walliser (1998). In the same area Becker et al. (2013) found only basal Devonian conodonts from this unit at El Khraouia, whereas Kröger (2008) draw the boundary on the upper part of the Scyphocrinites limestones at Filon Douze, on the basis of similitude of this section with the nearby localities studied by Hollard (1977) and Haude \& Walliser (1998). More to the east, at Ouidane Chebbi, Belka et al. (1999) referred the Scyphocrinites beds to the Lochkovian only.

Finally, a precise age of the Scyphocrinites Limestone in the Tafilalt was defined as spanning the Silurian/Devonian boundary in a preliminary note by Corriga et al. (2013, 2014). These author also demonstrated the stratigraphic range of the two types of lobolites and of the crinoidal crowns associated: the more primitive cirrus loboliths and genera Scyphocrinites and Carolicrinus occur in the whole Př́idolí, whereas the advanced plate loboliths and genera Camarocrinus and Marhoumacrinus are present in the top Přídolí beds (Upper detortus conodont Zone) and in the basal Lochkovian (Corriga et al. 2014). 


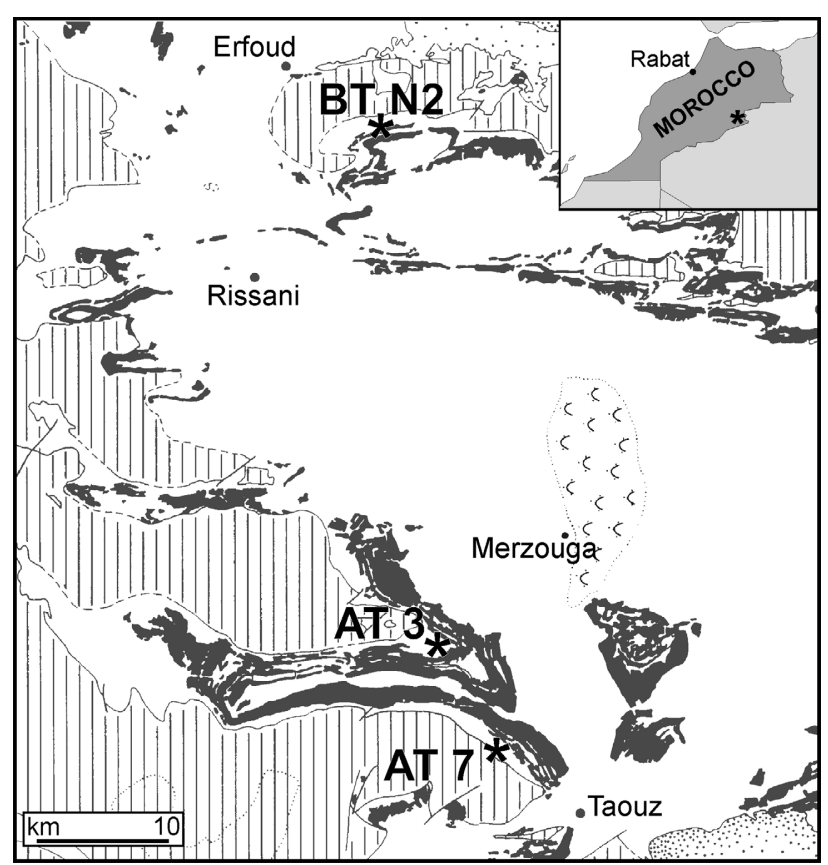

Figure 1. Location map.

Corriga et al. $(2013,2014)$ limited their investigation to the biostratigraphy of Scyphocrinites beds. In this paper the conodont association from the same three sections, from the Orthoceras limestones of Ludlow to the middle Lochkovian, is presented and discussed.

\section{Geological settings}

In the Tafilalt Silurian and lower-middle Lochkovian limestones are represented by carbonatic beds and lenses interbedded within thick shaley sequences, and forms more or less recognizable ridges (Haude \& Walliser 1998). These limestones mainly crop out in three distinct parts of the sequence.

The older limestones are represented by the classical "Orthoceras limestones" of Ludfordian age (Hollard 1977). They are mainly represented by a dark pelagic mudstone rich in orthoceratid cephalopods, with bivalves (Cardiola) and gastropods. Kröger (2008) named these limestones "Temperoceras Limestone", after the name of the more common cephalopod genus.

The "Scyphocrinites limestones" (Hollard 1977) occur across the Silurian-Devonian transition and are represented by several beds and lenses of centimetric to decimetric thickness. The majority of the limestone levels consists of crinoidal detritus, whereas others are micritic with parallely arranged nautiloids. In some layers larger parts of scyphocrinoids are present, locally with excellently preserved crowns on the lower surface (Haude \& Walliser 1998). Loboliths are always abundant.
A third group of limestones occur around the middle Lochkovian, and are mainly represented by orthoceratid limestones. However, if compared with the "Orthoceras limestones" and the "Scyphocrinites limestones" these middle Lochkovian beds are by far less widespread and documented. According to Rytina et al. (2013), these limestones can be correlated with bed PK of Kröger (2008).

\section{The studied sections}

Three sections, respectively named Bou Tchrafine N2 (loc. 474 of Walliser's locality catalogue), Atrous 3 (loc. 477) and Atrous 7 (loc. 540C) have been investigated (Fig. 1). Since the sections are very long and only the limestone beds are clearly visible in the field within long not exposed intervals, the thickness of the section is interpolated from the general inclination of exposed beds related to their horizontal distances, which were measured.

The Bou Tchrafine N2 section is located in the northern Tafilalt at the base of the northern side of Bou Tchrafine ridge, a few $\mathrm{km}$ southeast of Erfoud. The section is about $140 \mathrm{~m}$ thick (Fig. 2) and starts with a few Orthoceras Limestone beds and lenses cropping out along the plain. The Scyphocrinites beds here form evident small ridges and have a total thickness of about $21 \mathrm{~m}$.

The Atrous 3 and Atrous 7 sections are located in southern Tafilalt, northwest of Taouz. Atrous 3 is located slightly west of Chaib-er-Ras. Here about $70 \mathrm{~m}$ of $S c y$ phocrinites beds have been sampled (Fig. 3). In the lower part the limestones are scattered within the shales, whereas several beds crop out in the upper part of the section.

The Atrous 7 section (Fig. 4) is located SE of Bou Faddouz. According to Walliser notes, the section is probably very close to the section named "coupe de Taouz" by Hollard (1977). It starts with some beds of Orthoceras limestones, which yielded a rich fauna of middle Ludlow age. Following a long interval without exposures, the Scyphocrinites beds have a thickness limited to about 7 meters, definitely less than in the other sections studied. According to Walliser's sketch drawing of the section, sample AT 716 or AT 720 is equivalent to sample TM 720 by Hollard (1977). After a covered interval more than $100 \mathrm{~m}$ thick where only some dolerites are clearly visible, two beds of Orthoceras Limestone crop out: these beds yielded a mid-Lochkovian fauna.

\section{Conodont fauna}

More than fifty conodont samples, each between $2-3 \mathrm{~kg}$, were collected and processed with conventional acetic and/or formic acid technique, yielding about 2000 conodont elements (Tables 1-3). In general the state of preser- 


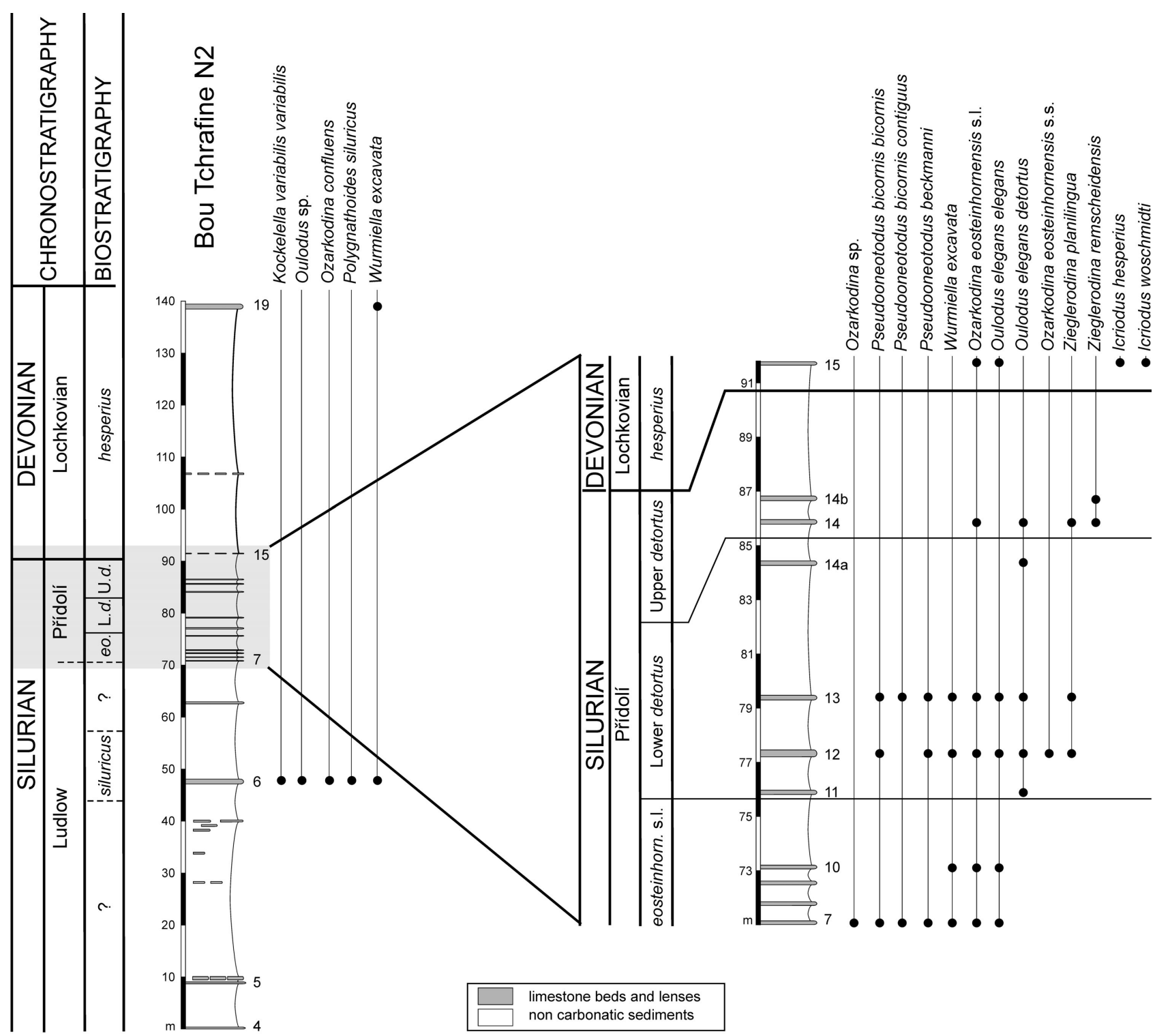

Figure 2. Stratigraphic log of the Bou Tchrafine N2 section, with occurrence of conodont species.

vation is quite good, even if better in Atrous 3 and Atrous 7, than in Bou Tchrafine N2 section. The abundance is higher in the Silurian than in the Devonian part of the sections, but differences in abundance may occur from bed to bed, and a few levels resulted barren. Color of conodonts is dark brown (Color Alteration Index $=3-4$ ), and is slightly darker in Bou Tchrafine than in Atrous sections.

The association includes 29 taxa belonging to 13 genera (Ancyrodelloides, Belodella, Dvorakia, Icriodus, Kockelella, Lanea, Oulodus, Ozarkodina, Pelekysgnathus, Polygnathoides, Pseudooneotodus, Wurmiella, Zieglerodina).

In the Ludlow the association is dominated by Kockelella variabilis variabilis, Polygnathoides siluricus and Wurmiella excavata. In Přídolí and lower Lochkovian strata Oulodus and ozarkodinids (mainly Zieglerodina and
"Ozarkodina") are dominant, whereas other genera are always scarce. Middle Lochkovian associations of Atrous 7 section are dominated by Pelekysgnathus and Ancyrodelloides. In general coniforms are always rare and present only in some levels with genera Dvorakia and Pseudooneotodus.

\section{Anomalous elements}

A few anomalous elements have been collected throughout the sections. They are mainly represented by ramiform elements with an unusually branched process. In literature such forms have been documented from several Silurian levels, and are particularly common in the siluricus Zone 


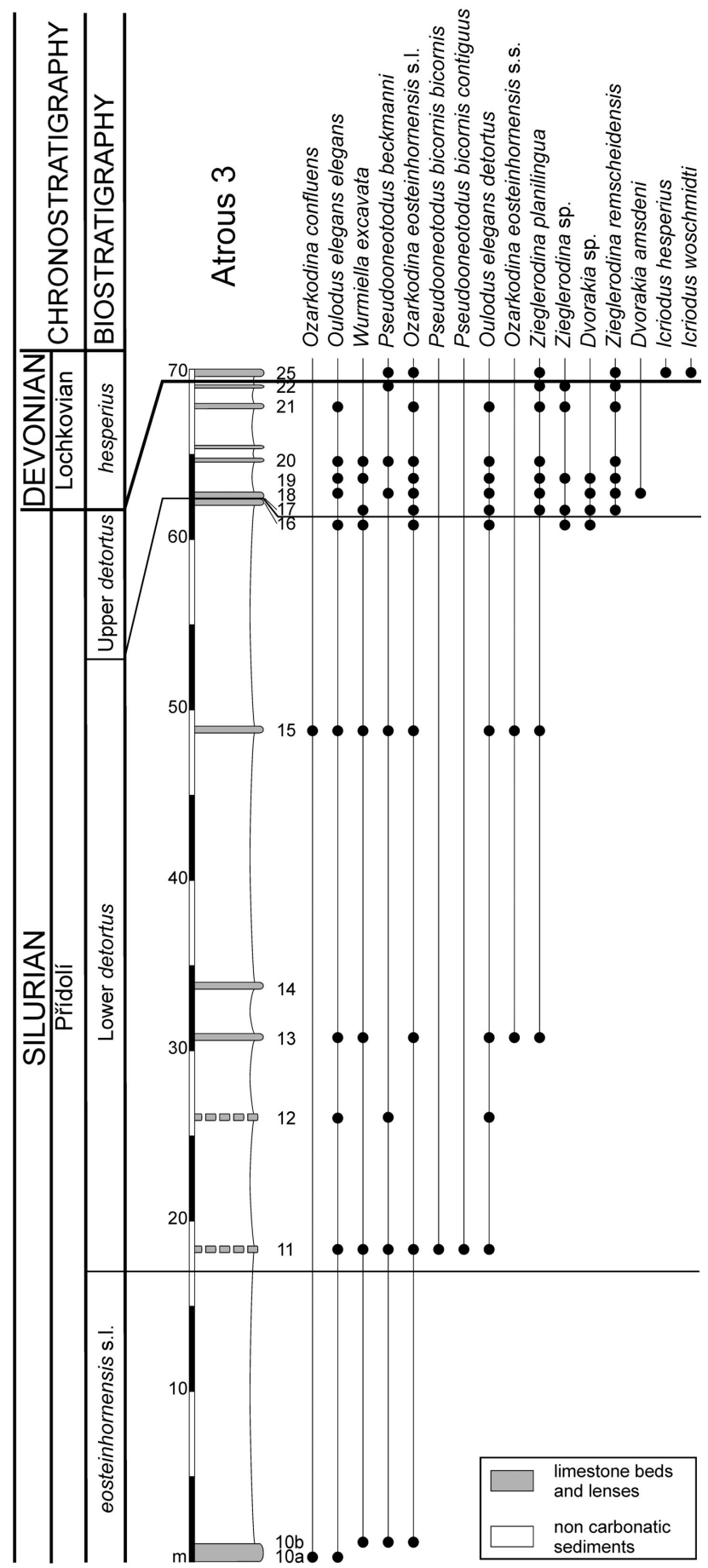

Figure 3. Stratigraphic log of the Atrous 3 section, with occurrence of conodont species.

(Klapper \& Murphy 1975; Corradini et al. 1996, 2009a, 2009b; Corriga et al. 2009; Slavík et al. 2010); beside, have been reported also from the top Llandovery amorphognathoides Zone (Walliser 1964, Helfrich 1980) and around the Silurian/Devonian boundary (Walliser 1964, Corradini \& Corriga 2010).
In the collections from Tafilalt, anomalously branched elements have been reported from the siluricus Zone (Fig. 5N) and from the Lochkovian transitans Zone (Fig. 5M). As far as we know, this is the first report of such forms from the Lower Devonian.

Beside these branched forms, in bed AT 3 10b (eosteinhornensis s.1. i.Z.) a deformed S0 element of Oulodus sp. has been found (Fig. 6R): on the left side the normal denticulation is present, whereas on the right process a few small denticles looks to have been grown above an older fracture surface.

\section{Biostratigraphy}

The biozonation schemes followed in this paper are those proposed by Cramer et al. (2011), for the Ludlow, and by Corradini \& Corriga (2012) for the Přídolí and Lochkovian.

The conodont faunas allow recognition of seven conodont zones in the studied sections: ploeckensis and siluricus zones in the Ludlow; eosteinhornensis s.1., Lower detortus and Upper detortus zones in the Přídolí; hesperius and transitans zones in the Lochkovian. A few intervals have not been documented, probably corresponding to the long shaley intervals, mostly covered, above and below the Scyphocrinites beds. In fact, the upper Ludlow snajdri interval Zone and crispa Zone, and the Lochkovian postwoschmidti and carlsi zones are missing.

\section{The ploeckensis Zone}

The ploeckensis Zone is possibly present at the base of the Atrous 7 section, in bed 11. The marker is missing, but the association of various species of Kockelella (with $K$. v. variabilis very abundant), Pseudoonetodus and Wurmiella excavata suggests a "pre-siluricus" interval. The biostratigraphic attribution is confirmed by the entry of Polygnathoides siluricus immediately above. However, a slightly older age (i.e. variabilis interval Zone) cannot be excluded.

\section{The siluricus Zone}

The occurrence of the marker Polygnathoides siluricus allows to discriminate the siluricus Zone in Bou Tchrafine (bed 6) and Atrous 7 (beds 12-13) sections. Pol. siluricus and Wurmiella excavata are dominant; Kockelella v. variabilis and $K . v$. ichnusae are present only in the lower part of the zone, confirming their known range from other part of the world (Serpagli \& Corradini 1999; Slavík et al. 2010, 2013). 


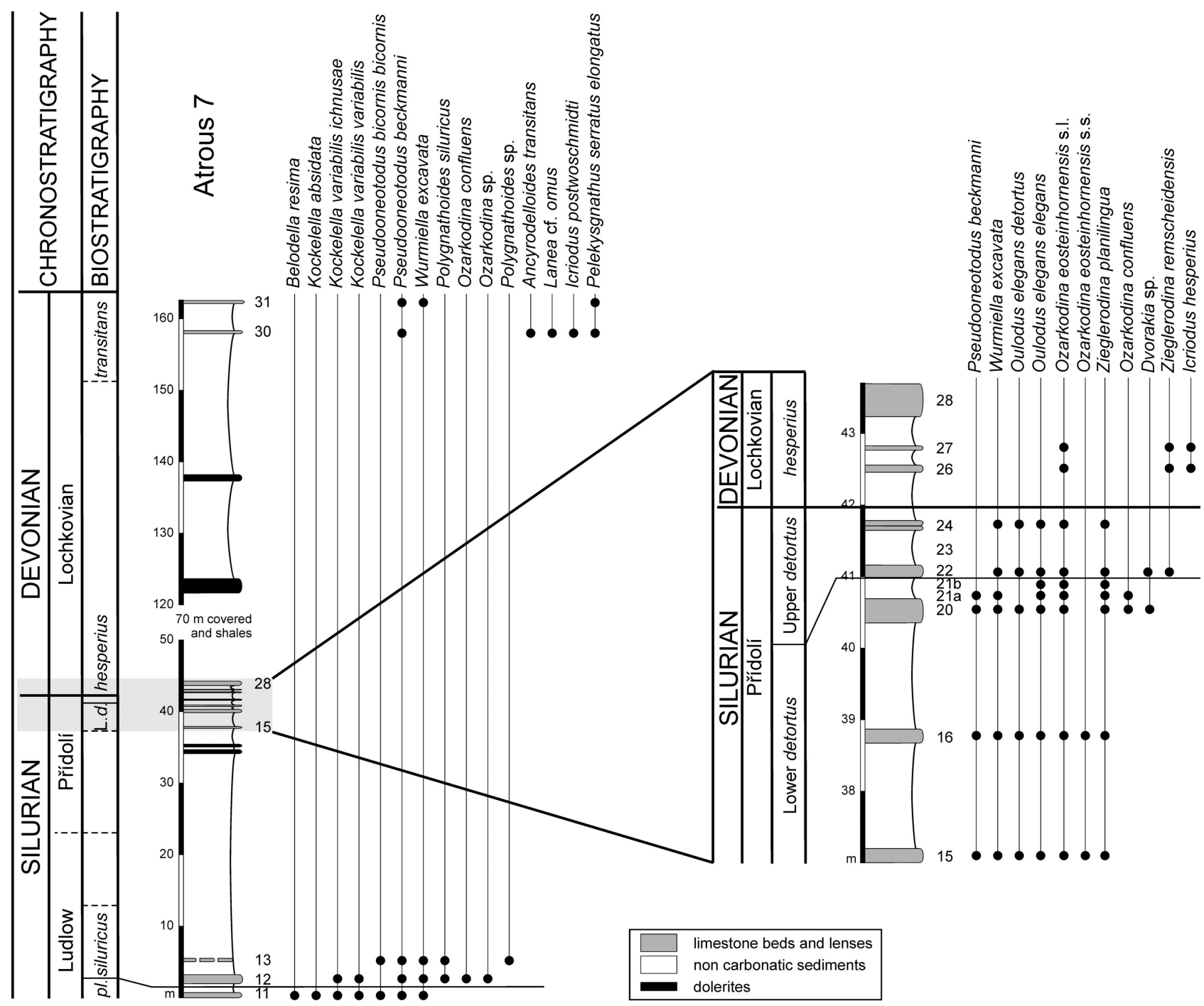

Figure 4. Stratigraphic log of the Atrous 7 section, with occurrence of conodont species.

\section{The eosteinhornensis s.I. interval Zone}

We attribute to this zone beds 7-10 of Bou Tchrafine N2 section and beds $10 \mathrm{a}-10 \mathrm{~b}$ of Atrous 3 section, due to the occurrence of a typical lower Př́dolí association, below the entry of Oulodus el. detortus. In general, conodonts are not abundant in this interval; the association is dominated by Pseudooneotodus, whereas Oulodus elegans elegans is common.

\section{The Lower detortus Zone}

The Lower detortus Zone is discriminated by the entry of the marker Oulodus el. detortus in the three sections: Bou Tchrafine (beds 11-14a), Atrous 3 (beds 11-16) and Atrous 7 (beds 15-21b) sections. The "Ozarkodina" eosteinhornensis s.s. horizon is present in all the sections, and
Zieglerodina planilingua enters within this horizon. Oulodus el. detortus, Oul. el. elegans and "Ozarkodina" eosteinhornensis s.l. are dominant in this interval.

\section{The Upper detortus Zone}

The Upper detortus Zone is recognized in Bou Tchrafine (beds 14-14b), Atrous 3 (beds 17-22) and Atrous 7 (beds 22-24) sections by the first occurrence of Zieglerodina remscheidensis that enters within this zone (Corradini \& Corriga 2012). In fact, the lower boundary of the zone is defined by the extinction of Dapsilodus obliquicostatus, but neither this taxon, nor Coryssognathus dubius or Panderodus recuvatus that have the same last occurrence (Corradini \& Corriga 2012) have been found in the studied section. Therefore, the lower boundary of this zone can be in a slightly lower position in all the three sections. 
Bulletin of Geosciences • Vol. 89, 1, 2014

Table 1. Distribution of conodonts in the Bou Tchrafine N2 section.

\begin{tabular}{|c|c|c|c|c|c|c|c|c|c|c|c|c|c|c|c|c|c|c|c|c|}
\hline bed & & 1 & 2 & 3 & 5 & 6 & 7 & 10 & 11 & 12 & 12 & 12 & 12 & 13 & $14 \mathrm{a}$ & $1414 b$ & 15 & $15 \mathrm{a}$ & 19 & 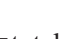 \\
\hline sample & & 3738 & 739 & 740 & 3741 & $3742 ?$ & 742 & 3790 & 3791 & 3792 & 3744 & 3745 & 3746 & 3747 & 73793 & 37483798 & 3794 & 3749 & 3750 & )$^{\text {total }}$ \\
\hline \multirow[t]{2}{*}{ Icriodus hesperius } & P1 & & & & & & & & & & & & & & & & 3 & & & 3 \\
\hline & M & & & & & & & & & & & & & & & & 3 & & & 3 \\
\hline \multirow[t]{2}{*}{ Icriodus woschmidti } & $\mathrm{P} 1$ & & & & & & & & & & & & & & & & & 4 & & 4 \\
\hline & $\mathrm{M}$ & & & & & & & & & & & & & & & & & 2 & & 2 \\
\hline \multirow[t]{4}{*}{ Kockelella variabilis variabilis } & $\mathrm{P} 1$ & & & & & 2 & & & & & & & & & & & & & & 2 \\
\hline & So & & & & & 1 & & & & & & & & & & & & & & 1 \\
\hline & $\mathrm{S} 1$ & & & & & 1 & & & & & & & & & & & & & & 1 \\
\hline & $\mathrm{S} 2$ & & & & & 4 & & & & & & & & & & & & & & 4 \\
\hline \multirow[t]{6}{*}{ Oulodus elegans detortus } & $\mathrm{P} 1$ & & & & & & & & & & & & & 9 & 9 & & & & & 9 \\
\hline & $\mathrm{P} 2$ & & & & & & & & & & & & & 3 & 3 & & & & & 3 \\
\hline & M & & & & & & & & & & 3 & 1 & 22 & 3 & 3 & & & & & 29 \\
\hline & So & & & & & & & & 1 & & & & 3 & & & & & & & 4 \\
\hline & $\mathrm{S} 1$ & & & & & & & & & & & & 2 & 1 & 1 & & & & & 3 \\
\hline & $\mathrm{S} 2$ & & & & & & & & 1 & & 7 & 3 & 36 & 4 & 4 & 1 & & & & 52 \\
\hline \multirow[t]{5}{*}{ Oulodus elegans elegans } & $\mathrm{P} 2$ & & & & & & & 3 & & & 5 & & 11 & & & & & & & 19 \\
\hline & $\mathrm{M}$ & & & & & & & 1 & & & & 1 & 14 & & 1 & & & & & 17 \\
\hline & So & & & & & & & & & & 3 & & 2 & 1 & 1 & & & & & 6 \\
\hline & $\mathrm{S} 1$ & & & & & & & & & & 1 & & 5 & 1 & 1 & & & 2 & & 9 \\
\hline & $\mathrm{S} 2$ & & & & & & 1 & 2 & & & & & 1 & 4 & 4 & & & & & 8 \\
\hline \multirow[t]{2}{*}{ Oulodus sp. } & $\mathrm{S} 1$ & & & & & 1 & & & & & & & & & & & & & & $\overline{1}$ \\
\hline & $\mathrm{S} 2$ & & & & & 4 & & & & & & & & & & & & & & 4 \\
\hline Ozarkodina confluens & $\mathrm{P} 1$ & & & & & 1 & & & & & & & & & & & & & & 1 \\
\hline Ozarkodina eosteinhornensis s.s. & $\mathrm{P} 1$ & & & & & & & & & & 1 & & 5 & & & & & & & 6 \\
\hline \multirow[t]{6}{*}{ Ozarkodina eosteinhornensis s.1. } & $\mathrm{P} 1$ & & & & & & 2 & 1 & & & 2 & 2 & 14 & 1 & 1 & 1 & 5 & 1 & & 29 \\
\hline & $\mathrm{P} 2$ & & & & & & & & & & 2 & 2 & 5 & & & & 1 & 1 & & 11 \\
\hline & M & & & & & & & & & & & 1 & 1 & & & & & & & 2 \\
\hline & So & & & & & & & & & & & & 1 & & & & & & & 1 \\
\hline & $\mathrm{S} 1$ & & & & & & & & & & 1 & & & & & & & & & 1 \\
\hline & $\mathrm{S} 2$ & & & & & & & 1 & & & & 1 & 2 & & & & 1 & & & 5 \\
\hline Ozarkodina sp. & $\mathrm{P} 1$ & & & & & & 2 & & & & & & & & & & & & & 2 \\
\hline \multirow[t]{3}{*}{ Polygnathoides siluricus } & $\mathrm{P} 1$ & & & & & 2 & & & & & & & & & & & & & & 2 \\
\hline & M & & & & & 1 & & & & & & & & & & & & & & 1 \\
\hline & $\mathrm{S} 1$ & & & & & 1 & & & & & & & & & & & & & & 1 \\
\hline Pseudooneotodus beckmanni & & & & & & & 4 & & & & & & 16 & 6 & 6 & & & 8 & & 34 \\
\hline $\begin{array}{l}\text { Pseudooneotodus bicornis } \\
\text { contiguus }\end{array}$ & & & & & & & 9 & & & & & & & 2 & 2 & & & & & 11 \\
\hline $\begin{array}{l}\text { Pseudooneotodus bicornis } \\
\text { bicornis }\end{array}$ & & & & & & & 38 & & & & & & 1 & 1 & 1 & & & & & 40 \\
\hline \multirow[t]{5}{*}{ Wurmiella excavata } & $\mathrm{P} 1$ & & & & & 7 & 1 & 1 & & & 1 & 2 & 2 & 1 & 1 & & & & & 15 \\
\hline & $\mathrm{P} 2$ & & & & & 1 & & & & & & & 2 & & & & & & 1 & 4 \\
\hline & S0 & & & & & & & & & & 1 & & & & & & & & & 1 \\
\hline & $\mathrm{S} 1$ & & & & & & 1 & & & & & & 1 & & & & & & & 2 \\
\hline & $\mathrm{S} 2$ & & & & & 2 & 1 & & & & & & & & & & & & & 3 \\
\hline Zieglerodina planilingua & $\mathrm{P} 1$ & & & & & & & & & & & 3 & 2 & 5 & 5 & 2 & & & & 12 \\
\hline \multirow[t]{3}{*}{ Zieglerodina remscheidensis } & $\mathrm{P} 1$ & & & & & & & & & & & & & & & 1 & & & & 1 \\
\hline & $\mathrm{P} 2$ & & & & & & & & & & & & & & & 1 & & & & 1 \\
\hline & $\mathrm{S} 2$ & & & & & & & & & & & & & & & 1 & & & & 1 \\
\hline Indetermined and fragments & & & & & & 8 & 3 & 3 & & & & 3 & 32 & 5 & 5 & & & & & 54 \\
\hline Total & & 0 & 0 & 0 & 0 & 36 & 62 & 12 & 2 & 0 & 27 & 19 & 180 & 47 & 1 & 7 & 13 & 18 & & 425 \\
\hline
\end{tabular}


Table 2. Distribution of conodonts in the Atrous 3 section.

\begin{tabular}{|c|c|c|c|c|c|c|c|c|c|c|c|c|c|c|c|c|c|c|c|c|c|}
\hline bed & & $10 \mathrm{a}$ & $10 \mathrm{~b}$ & 11 & 12 & 13 & 14 & 15 & 16 & 17 & $17 \mathrm{~b}$ & $17 \mathrm{c}$ & 18 & 19 & 19 & 20 & 20 & 21 & 22 & 25 & $-t a t a l$ \\
\hline sample & & 3707 & 3708 & 3709 & 3710 & 3711 & 3712 & 3713 & 3714 & 3787 & 3716 & 3717 & 3718 & 3788 & 3719 & 3720 & 3789 & 3721 & 3722 & 3723 & \\
\hline Dvorakia amsdeni & S3 & & & & & & & & & & & & 1 & & & & & & & & 1 \\
\hline Dvorakia sp. & & & & & & & & & 2 & 4 & 3 & & 1 & 1 & 5 & & & & & & 16 \\
\hline Icriodus hesperius & $\mathrm{P} 1$ & & & & & & & & & & & & & & & & & & & 3 & 3 \\
\hline & M & & & & & & & & & & & & & & & & & & & 4 & 4 \\
\hline Icriodus woschmidti & $\mathrm{P} 1$ & & & & & & & & & & & & & & & & & & & 6 & 6 \\
\hline & $\mathrm{M}$ & & & & & & & & & & & & & & & & & & & 1 & 1 \\
\hline Oulodus elegans detortus & $\mathrm{P} 1$ & & & & 1 & & & & 2 & & & & & & & & & & & & 3 \\
\hline & $\mathrm{P} 2$ & & & & 1 & & & 2 & & & 1 & & 1 & & & 4 & 1 & & & & 10 \\
\hline & M & & & & & 5 & & 30 & & 9 & & 2 & 1 & & 1 & 4 & & 1 & & & 53 \\
\hline & So & & & & & 1 & & 1 & 1 & & & 1 & 5 & 1 & 1 & & & & & & 11 \\
\hline & $\mathrm{S} 1$ & & & & 3 & & & 7 & & & & & 3 & & 4 & 1 & & 8 & & & 26 \\
\hline & $\mathrm{S} 2$ & & & 1 & 1 & 6 & & 11 & 7 & 5 & 2 & 2 & 8 & 1 & 8 & & 9 & 5 & & & 66 \\
\hline Oulodus elegans elegans & $\mathrm{P} 1$ & 1 & 5 & & 1 & 1 & & & & & 2 & 1 & & & & & & & & & 11 \\
\hline & $\mathrm{P} 2$ & & 5 & & 2 & 4 & & 3 & 15 & 3 & & & & & 1 & 2 & & 1 & & & 36 \\
\hline & M & 2 & 9 & 2 & 3 & 1 & & 8 & & 3 & 3 & 2 & 2 & & & 1 & 1 & 1 & & & 38 \\
\hline & So & 1 & 2 & 3 & 2 & 2 & & & 1 & & 4 & 1 & 4 & & 1 & & 1 & 1 & & & 23 \\
\hline & $\mathrm{S} 1$ & & 1 & & & & & 2 & & 3 & & 1 & & & 1 & & 2 & 2 & & & 12 \\
\hline & $\mathrm{S} 2$ & & 6 & 1 & & 3 & & 1 & 10 & 3 & & 2 & & & & 2 & & & & & 28 \\
\hline Ozarkodina confluens & $\mathrm{P} 1$ & 1 & & & & & & 1 & & & & & & & & & & & & & 2 \\
\hline & $\mathrm{P} 2$ & 1 & & & & & & & & & & & & & & & & & & & 1 \\
\hline $\begin{array}{l}\text { Ozarkodina } \\
\text { eosteinhornensis s.s. }\end{array}$ & $\mathrm{P} 1$ & & & & & 8 & & 1 & & & & & & & & & & & & & 9 \\
\hline Ozarkodina & $\mathrm{P} 1$ & & 4 & 2 & & 3 & & 31 & 7 & 9 & 8 & 8 & 21 & & 12 & 33 & 13 & 6 & 1 & 2 & 160 \\
\hline eosteinhornensis s.1. & $\mathrm{P} 2$ & & 1 & & & 4 & & 11 & & 15 & & 3 & 3 & & 6 & 20 & 1 & 3 & 1 & 1 & 69 \\
\hline & M & & & & & 1 & & 2 & & & 1 & 1 & & & 1 & & & 1 & & & 7 \\
\hline & S0 & & 1 & & & & & 2 & & 3 & 2 & & 1 & & & 1 & & 1 & & & 11 \\
\hline & $\mathrm{S} 1$ & & & & & 1 & & 1 & & & & & & & 9 & & & & 1 & & 12 \\
\hline & $\mathrm{S} 2$ & & & & & 2 & & 2 & & 3 & 2 & & 3 & & 6 & 3 & 1 & & 4 & & 26 \\
\hline Ozarkodina sp. & $\mathrm{P} 1$ & & & & & & & & & & & 3 & & & & & & & & & 3 \\
\hline $\begin{array}{l}\text { Pseudooneotodus } \\
\text { beckmanni }\end{array}$ & & & 12 & 11 & 3 & 1 & & 4 & & & & & 1 & & & 1 & & & 2 & 5 & 40 \\
\hline $\begin{array}{l}\text { Pseudooneotodus bicornis } \\
\text { bicornis }\end{array}$ & & & & 1 & & & & & & & & & & & & & & & & & 1 \\
\hline $\begin{array}{l}\text { Pseudooneotodus bicornis } \\
\text { contiguus }\end{array}$ & & & & 1 & & & & & & & & & & & & & & & & & 1 \\
\hline Wurmiella excavata & $\mathrm{P} 1$ & & 1 & 1 & & 1 & & 1 & 24 & 1 & & & & & 1 & 9 & 1 & & & & 40 \\
\hline & $\mathrm{P} 2$ & & & & & & & & & & & & & & & 10 & & & & & 10 \\
\hline & M & & & & & & & & & & & & & & & 1 & & & & & 1 \\
\hline & S0 & & & & & & & & 1 & & & & & & & 5 & & & & & 6 \\
\hline & $\mathrm{S} 1$ & & & & & & & & & & & & & & & 4 & & & & & 4 \\
\hline & $\mathrm{S} 2$ & & & & & & & & 1 & & & & & & & 2 & & & & & 3 \\
\hline Zieglerodina planilingua & $\mathrm{P} 1$ & & & & & 2 & & 2 & & 2 & 4 & 5 & 7 & & 13 & 2 & 7 & 13 & 7 & 1 & 65 \\
\hline & $\mathrm{P} 2$ & & & & & & & & & 1 & & & 3 & & 2 & & & 1 & & & 7 \\
\hline & $\mathrm{M}$ & & & & & & & & & & & & 1 & & 1 & & & & 2 & & 4 \\
\hline & $\mathrm{S} 1$ & & & & & & & & & & & & 1 & & 2 & & & & & & 3 \\
\hline Zieglerodina & $\mathrm{P} 1$ & & & & & & & & & & 2 & 2 & 10 & & 4 & 3 & & 2 & 2 & 2 & 27 \\
\hline remscheidensis & $\mathrm{P} 2$ & & & & & & & & & & & & 4 & & & & & & & & 4 \\
\hline & $\mathrm{S} 1$ & & & & & & & & & & & & & & & 1 & & & & & 1 \\
\hline Zieglerodina sp. & $\mathrm{P} 1$ & & & & & & & & 1 & & 1 & & & & 2 & & & 1 & 1 & & 6 \\
\hline Fragments & & 2 & 3 & 4 & 3 & 2 & 1 & 4 & 5 & 2 & 2 & 1 & 2 & 1 & 3 & 2 & 2 & 1 & 1 & 2 & 43 \\
\hline Total & & 8 & 50 & 27 & 20 & 48 & 1 & 127 & 77 & 66 & 37 & 35 & 83 & 4 & 84 & 111 & 39 & 48 & 22 & 27 & 914 \\
\hline
\end{tabular}


Bulletin of Geosciences • Vol. 89, 1, 2014

Table 3. Distribution of conodonts in the Atrous 7 section.

\begin{tabular}{|c|c|c|c|c|c|c|c|c|c|c|c|c|c|c|c|c|c|c|}
\hline bed & & 11 & 12 & 13 & 15 & 16 & 20 & $21 \mathrm{a}$ & $21 b$ & 22 & 23 & 24 & 26 & 27 & 28 & 30 & 31 & total \\
\hline sample & & 3724 & 3725 & 3726 & 3727 & 3728 & 3729 & 3796 & 3795 & 3730 & 3797 & 3731 & 3732 & 3733 & 3734 & 3735 & 3736 & \\
\hline Ancyrodelloides transitans & $\mathrm{P} 1$ & & & & & & & & & & & & & & & 5 & & 5 \\
\hline Belodella resima & $\mathrm{Sa}$ & 1 & & & & & & & & & & & & & & & & 1 \\
\hline Dvorakia sp. & & & & & & & 1 & & & 4 & & & & & & & & 5 \\
\hline \multirow{2}{*}{ Icriodus hesperius } & $\mathrm{P} 1$ & & & & & & & & & & & & 4 & 34 & & & & 38 \\
\hline & $\mathrm{M}$ & & & & & & & & & & & & & 8 & & & & 8 \\
\hline Icriodus postwoschmidti & $\mathrm{P} 1$ & & & & & & & & & & & & & & & 1 & & 1 \\
\hline Kockelella ortus absidata & $\mathrm{P} 1$ & 2 & & & & & & & & & & & & & & & & 2 \\
\hline Kockelella variabilis ichnusae & $\mathrm{P} 1$ & 2 & 3 & & & & & & & & & & & & & & & 5 \\
\hline \multirow[t]{5}{*}{ Kockelella variabilis variabilis } & $\mathrm{P} 1$ & 6 & 2 & & & & & & & & & & & & & & & 8 \\
\hline & $\mathrm{P} 2$ & 3 & 2 & & & & & & & & & & & & & & & 5 \\
\hline & S0 & & 5 & & & & & & & & & & & & & & & 5 \\
\hline & $\mathrm{S} 1$ & 3 & 4 & & & & & & & & & & & & & & & 7 \\
\hline & $\mathrm{S} 2$ & 1 & 4 & & & & & & & & & & & & & & & 5 \\
\hline \multirow[t]{2}{*}{ Lanea cf. omus } & $\mathrm{P} 1$ & & & & & & & & & & & & & & & 1 & & 1 \\
\hline & $\mathrm{P} 2$ & & & & & & & & & & & & & & & 1 & & 1 \\
\hline \multirow[t]{6}{*}{ Oulodus elegans detortus } & $\mathrm{P} 1$ & & & & & & & & & 1 & & & & & & & & 1 \\
\hline & $\mathrm{P} 2$ & & & & 4 & 2 & 1 & & & 3 & & & & & & & & 10 \\
\hline & M & & & & 3 & 1 & 3 & & & 2 & & & & & & & & 9 \\
\hline & So & & & & 1 & & 2 & & & 2 & & 2 & & & & & & 7 \\
\hline & $\mathrm{S} 1$ & & & & 1 & & 2 & & & & & & & & & & & 3 \\
\hline & $\mathrm{S} 2$ & & & & 10 & 2 & 8 & & & 4 & & 2 & & & & & & 26 \\
\hline \multirow[t]{6}{*}{ Oulodus elegans elegans } & $\mathrm{P} 1$ & & & & 1 & & & & 1 & & & & & & & & & 2 \\
\hline & $\mathrm{P} 2$ & & & & 1 & 1 & 1 & & & & & & & & & & & 3 \\
\hline & M & & & & & 1 & 1 & 1 & & 1 & & 2 & & & & & & 6 \\
\hline & S0 & & & & 5 & & 1 & 1 & & 1 & & & & & & & & 8 \\
\hline & $\mathrm{S} 1$ & & & & & & 4 & & & 1 & & 1 & & & & & & 6 \\
\hline & $\mathrm{S} 2$ & & & & 3 & & 3 & & 1 & & & & & & & & & 7 \\
\hline \multirow[t]{2}{*}{ Ozarkodina confluens } & $\mathrm{P} 1$ & & 2 & & & & 10 & 2 & & & & & & & & & & 14 \\
\hline & $\mathrm{S} 2$ & & 1 & & & & & & & & & & & & & & & 1 \\
\hline Ozarkodina eosteinhornensis s.s. & $\mathrm{P} 1$ & & & & 1 & 2 & & & & & & & & & & & & 3 \\
\hline \multirow[t]{6}{*}{ Ozarkodina eosteinhornensis s.1. } & $\mathrm{P} 1$ & & & & 13 & 3 & 9 & 2 & 2 & 6 & & 18 & 2 & 5 & & & & 60 \\
\hline & $\mathrm{P} 2$ & & & & 1 & 1 & 1 & 1 & & 1 & & 2 & & & & & & 7 \\
\hline & M & & & & & & & & & & & 1 & & & & & & 1 \\
\hline & So & & & & & & 1 & & & 1 & & 1 & & & & & & 3 \\
\hline & $\mathrm{S} 1$ & & & & & & & & & 1 & & & & & & & & 1 \\
\hline & $\mathrm{S} 2$ & & & & 1 & & 2 & 2 & & & & & & & & & & 5 \\
\hline \multirow[t]{2}{*}{ Ozarkodina sp. } & $\mathrm{P} 1$ & & 1 & & & & & & & & & & & & & & & 1 \\
\hline & $\mathrm{P} 2$ & & 1 & & & & & & & & & & & & & & & 1 \\
\hline \multirow[t]{2}{*}{ Pelekysgnatus serratus elongatus } & $\mathrm{P} 1$ & & & & & & & & & & & & & & & 19 & 1 & 20 \\
\hline & M & & & & & & & & & & & & & & & 2 & 2 & 4 \\
\hline \multirow[t]{6}{*}{ Polygnathoides siluricus } & $\mathrm{P} 1$ & & 12 & 8 & & & & & & & & & & & & & & 20 \\
\hline & $\mathrm{P} 2$ & & 9 & 3 & & & & & & & & & & & & & & 12 \\
\hline & M & & 3 & & & & & & & & & & & & & & & 3 \\
\hline & S0 & & & 1 & & & & & & & & & & & & & & 1 \\
\hline & $\mathrm{S} 1$ & & 1 & & & & & & & & & & & & & & & 1 \\
\hline & $\mathrm{S} 2$ & & 2 & 2 & & & & & & & & & & & & & & 4 \\
\hline Polygnathoides sp. & $\mathrm{P} 1$ & & & 1 & & & & & & & & & & & & & & 1 \\
\hline Pseudooneotodus beckmanni & & 1 & 1 & 7 & 1 & 1 & 1 & & & & & & & & & 1 & 6 & 19 \\
\hline
\end{tabular}


Maria G. Corriga et al. • Upper Silurian and Lower Devonian conodonts from southeastern Morocco

\begin{tabular}{|c|c|c|c|c|c|c|c|c|c|c|c|c|c|c|c|c|c|c|}
\hline bed & & 11 & 12 & 13 & 15 & 16 & 20 & $21 \mathrm{a}$ & $21 b$ & 22 & 23 & 24 & 26 & 27 & 28 & 30 & 31 & total \\
\hline sample & & 3724 & 3725 & 3726 & 3727 & 3728 & 3729 & 3796 & 3795 & 3730 & 3797 & 3731 & 3732 & 3733 & 3734 & 3735 & 3736 & \\
\hline $\begin{array}{l}\text { Pseudooneotodus bicornis } \\
\text { bicornis }\end{array}$ & & 1 & & 12 & & & & & & & & & & & & & & 13 \\
\hline \multirow[t]{6}{*}{ Wurmiella excavata } & P1 & 3 & 28 & 3 & 1 & 1 & 10 & 2 & & 1 & & 1 & & & & & 1 & 51 \\
\hline & $\mathrm{P} 2$ & & 2 & 3 & & & & & & & & & & & & & & 5 \\
\hline & M & & 2 & 3 & & & & & & & & 1 & & & & & & 6 \\
\hline & So & & 1 & 1 & & & & & & & & 1 & & & & & & 3 \\
\hline & S1 & & 2 & 2 & & & & & & & & 1 & & & & & & 5 \\
\hline & S2 & & 8 & 8 & & & & & & & & 1 & & & & & & 17 \\
\hline \multirow[t]{2}{*}{ Zieglerodina planilingua } & $\mathrm{P} 1$ & & & & 1 & 4 & 1 & 8 & 3 & 20 & & 3 & & & & & & 40 \\
\hline & $\mathrm{P} 2$ & & & & & 1 & & 1 & & & & & & & & & & 2 \\
\hline \multirow[t]{2}{*}{ Zieglerodina remscheidensis } & $\mathrm{P} 1$ & & & & & & & & & 3 & & & 1 & 9 & & & & 13 \\
\hline & $\mathrm{P} 2$ & & & & & & & & & 1 & & & & & & & & 1 \\
\hline Zieglerodina sp. & P1 & & & & & & & 1 & & & & 1 & & & & & 1 & 3 \\
\hline Anomalous elements & & & 3 & & & & & & & & & & & & & 1 & & 4 \\
\hline Indetermined and fragments & & 6 & 15 & 17 & 4 & 8 & 2 & 4 & 1 & 5 & & 2 & 4 & 6 & & 5 & 2 & 81 \\
\hline Total & & 28 & 114 & 71 & 52 & 28 & 64 & 25 & 8 & 58 & 0 & 40 & 11 & 62 & 0 & 28 & 13 & 602 \\
\hline
\end{tabular}

In the Bou Tchrafine section conodonts are very rare in this interval. Oulodus (Oul. el. elegans and Oul. el. detortus) and "Ozarkodina" eosteinhornensis s.l. are always abundant, as well as Zieglerodina planilingua in some levels (i.e.: AT 3 19b, AT 7 22).

\section{The hesperius Zone}

The entry of the marker Icriodus hesperius allows discriminating the hesperius Zone in Atrous 3 (beds 25) and Atrous 7 (beds 26-18) sections. Icriodus is the dominant genus in this interval.

\section{The transitans Zone}

The transitans Zone is discriminated in the uppermost part of the Atrous 7 section (beds 30-31) by the occurrence of the marker Ancyrodelloides transitans and of Pelekysgnathus serratus elongatus. These forms are the only taxa abundant in these beds, while the other species collected are rare.

\section{Phylogenesis of genera Lanea and Ancyrodelloides}

Genus Lanea has been established by Murphy \& Valenzuela-Ríos (1999), subdividing species previously included in Ancyrodelloides and moving a few other taxa from other genera (i.e. "Spathognathodus" telleri). According the original diagnosis, Lanea is characterized by the evident "terrace", the unrestricted basal cavity and the normally unornamented basal platform. Representatives of "Ancyrodelloides have ridges or tubercles above one or both the basal platform lobes, whereas only rare variants of some of the derived members of Lanea have ridges or tubercles" and "the processes found in the Pa elements of Lanea are lobate, whereas those in Ancyrodelloides are extensiform" (Murphy \& Valenzuela-Ríos 1999, p. 327).

In the same paper Murphy \& Valenzuela-Ríos (1999) also erected three new species: "Ozarkodina" planilingua (now Zieglerodina planilingua), Lanea eoeleanorae and Lanea omoalpha. The first is considered as the ancestor of Lanea, having a small terrace. The latter, that is the former morphotype $\alpha$ of Ancyrodelloides omus Murphy \& Matti, 1983, is the older species of Lanea. Additionally, Murphy \& Valenzuela-Ríos (1999) assigned the species L. eleanorae (Lane \& Ormiston, 1979) and L. telleri (Schulze, $1968)$ to their new genus Lanea.

Murphy \& Valenzuela-Ríos (1999) published also a list of taxa left to Ancyrodelloides: A. trigonicus Bischoff \& Sannemann, 1958, A. transitans (Bischoff \& Sannemann, 1958), A. kutscheri Bischoff \& Sannemann, 1958, A. asymmetricus (Bischoff \& Sannemann, 1958), A. carlsi (Boersma, 1973), A. omus Murphy \& Matti, 1983, A. orcula Wilson, 1989, A. secus Barrick \& Klapper, 1992, A. cruzae Valenzuela-Ríos, 1994 and A. murphyi Valenzuela-Ríos, 1994. Later, A. sequeirosi Valenzuela-Ríos, 1999 has been established.

Recently Slavík (2011) moved A. carlsi to Lanea and suggested that also A. omus $\beta$ morph Murphy \& Matti, 1983 should be attributed to Lanea on the basis of the shape of the basal cavity and the presence of some ornamentation on the platform. We agree that A. omus should be attributed to Lanea for the strong affinity with L. omoalpha, from 
which it differs mainly by the occurrence of denticles on the platform terraces: the shape of the lobes, the wide terraces and the lacking of strong ornamentation on the platform are characteristic more close to Lanea, than to Ancyrodelloides. Therefore the taxon should be named $L$. omus (Murphy \& Matti 1983).

On the other hand, we disagree on the new attribution of A. carlsi to Lanea: the species was described as Spathognathodus carlsi by Boersma (1973) and moved to Ancyrodelloides by Klapper (in Ziegler 1991). In our opinion the well-developed asymmetrical lateral process, without a well evident terrace, bearing strong denticles, or even nodes, is a characteristic of Ancyrodelloides, and therefore it looks more appropriate leave it in this genus. However, other characteristics, like the wide-open basal cavity are more typical and fit better in Lanea. It should be pointed out that the diagnoses of Ancyrodelloides and Lanea are not clear and overlap each other: the correct attribution of species to one or the other genus shall be defined only after a revision of the diagnoses based not only on the P1 element, but also on the complete apparatus. Up to now the only complete known apparatus (Slavík 2011) belongs to $A$. carlsi (named by the author L. carlsi), and the species shall be attributed certainly to one or the other genus only when the apparatuses of other species of Ancyrodelloides and Lanea will be reconstructed.

In the last years, several papers on Lochkovian conodonts have been published (i.e. Barrick et al. 2005; Corriga 2011; Corriga et al. 2011, 2012; Corradini \& Corriga 2012; Slavík et al. 2012; Drygant \& Szaniawski 2012; Mavrinskaya \& Slavík 2013), presenting data that can help in the reconstruction of the phylogenesis of Lanea and Ancyrodelloides (Fig. 7).

As already proposed by Murphy \& Valenzuela-Ríos (1999), genus Lanea originated from Zieglerodina planilingua around the base of the postwoschmidti Zone, when the first occurrence of L. omoalpha is recorded (see discussion in Corradini \& Corriga, 2012, p. 647). The phylogenesis of Lanea is simple and the lineage omoalpha-eoeleanorae-eleanorae-telleri has been described by Murphy \& Valenzuela-Ríos (1999). Lanea omus (=Ancyrodelloides omus morph. $\beta$ Murphy \& Matti, 1983) originated from L. omoalpha by the development of tubercles on the platform at the base of the transitans Zone.

The older Ancyrodelloides, A. carlsi, branched from Lanea omoalpha, by the development of a strong, denticulated lateral process. A. carlsi gave rise to A. transitans, characterized by denticulated lateral processes on both sides of the element (Slavík 2011). A. secus is a short ranging species within the transitans Zone that originated from A. transitans by development of a "V-shaped pattern" of nodes on the outer platform (Barrick \& Klapper 1992). Ancyrodelloides has a large radiation in the upper part of the middle Lochkovian, when two main lineages, branched from A. transitans can be recognized (Valenzuela-Ríos 1994, 1999). The first, more developed and represented mainly by taxa with a wide geographical distribution, is represented by A. trigonicus - A. kutscheri-A. sequeirosi. The second, that up to now looks limited to part of North Gondwana, includes A. cruzae - A. murphy. Slavík et al. (2012) observed the increased number of different morphologies with various branching patterns of lateral processes. They considered it as radiation in terminal range

Figure 5. A - Kockelella variabilis ichnusae Serpagli \& Corradini, 1998; upper view of P1 element GZG 1612-540C-3724-1, sample AT 7 11, ploeckensis Zone. • B - Kockelella variabilis variabilis Walliser, 1957; upper view of P1 element GZG 1612-540C-3724-2, sample AT 7 11, ploeckensis Zone. • C - Kockelella variabilis variabilis Walliser, 1957; upper view of P1 element GZG 1612-540C-3724-3, sample AT 7 11, ploeckensis Zone. - D - Kockelella absidata absidata Barrick \& Klapper, 1976; lateral view of P1 element GZG 1612-540C-3724-4, sample AT 7 11, ploeckensis Zone. - E - Polygnathoides siluricus Branson \& Mehl, 1933; upper view of P1 element GZG 1612-540C-3725-1, sample AT 7 12, siluricus Zone. - F - Polygnathoides siluricus Branson \& Mehl, 1933; upper view of P1 element GZG 1612-540C-3726-2, sample AT 7 13, siluricus Zone. - G - Polygnathoides siluricus Branson \& Mehl, 1933; upper view of P1 element GZG 1612-540C-3726-1, sample AT 7 13, siluricus Zone. - H - Polygnathoides siluricus Branson \& Mehl, 1933; lateral view of P2 element GZG 1612-540C-3725-2, sample AT 7 12, siluricus Zone. - I - Polygnathoides siluricus Branson \& Mehl, 1933; lateral view of M element GZG 1612-540C-3725-3, sample AT 7 12, siluricus Zone. - J - Polygnathoides sp.; upper-lateral view of P1 element GZG 1612-540C-3726-3, sample AT 7 13, siluricus Zone. • K - Pseudooneotodus bicornis bicornis Drygant, 1974; upper view GZG 1612-474-3742-1, sample BT 7, eosteinhornensis s.1. Zone. • L - Pseudooneotodus beckmanni (Bischoff \& Sannemann, 1958); lateral view GZG 1612-474-3742-2, sample BT 7, eosteinhornensis s.1. Zone. • M - anomalous element; lateral view of S1 element GZG 1612-540C-3735-6, sample AT 7 30, transitans Zone. • N - anomalous element; lateral view of S1 element GZG 1612-540C-3725-4, sample AT 7 12, siluricus Zone. • O - Icriodus woschmidti Ziegler, 1960; lateral view of M element GZG 1612-477-3723-1, sample AT 3 25, hesperius Zone. - P - Icriodus woschmidti Ziegler, 1960; upper view of P1 element GZG 1612-477-3723-2, sample AT 3 25, hesperius Zone. • Q - Icriodus hesperius Klapper \& Murphy, 1975; upper view of P1 element GZG 1612-540C-3733-1, sample AT 7 27, hesperius Zone. • R - Icriodus hesperius Klapper \& Murphy, 1975; lateral view of M element GZG 1612-540C-3733-2, sample AT 7 27, hesperius Zone. • S - Pelekysgnathus serratus elongatus Carls \& Gandl, 1969; lateral view of P1 element GZG 1612-540C-3735-1; sample AT 7 30, transitans Zone. • T - Pelekysgnathus serratus elongatus Carls \& Gandl, 1969; upper view of P1 element GZG 1612-540C-3735-2; sample AT 7 30, transitans Zone. • U - Lanea cf. omus (Murphy \& Matti, 1983); upper view of P1 element GZG 1612-540C-3735-3, sample AT 7 30, transitans Zone. • V - Lanea cf. omus (Murphy \& Matti, 1983); lateral view of P2 element GZG 1612-540C-3735-4, sample AT 7 30, transitans Zone. • W - Icriodus postwoschmidti Mashkova, 1968; upper view GZG 1612-540C-3735-5 sample AT 730 , transitans Zone. $•$ X - Ancyrodelloides transitans (Bischoff \& Sannemann, 1958); upper view of P1 element GZG 1612-540C-3735-6, sample AT 7 30, transitans Zone. 


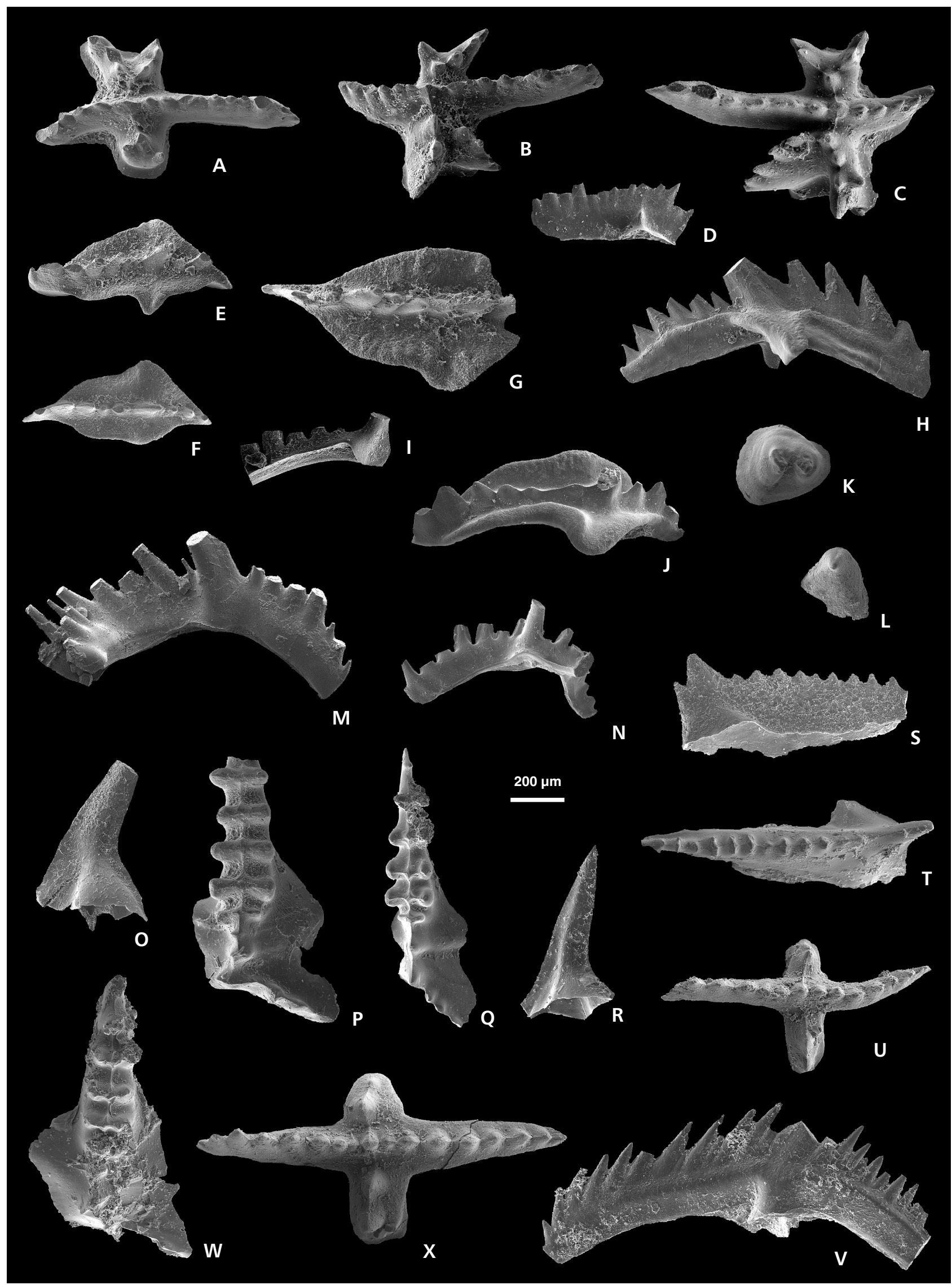


of Ancyrodelloides because ranges of these undescribed or formally described (but based on a very small number of specimens) forms are coeval.

More problematic is the connection of A. asymmetricus A. orcula with a defined species of the Ancyrodelloides stock. On the basis of the stratigraphical distribution of known taxa, A. asymmetricus can be originated only from A. transitans, by the loss of the outer lateral process. This relation was already suggested by Murphy \& Cebecioglu (1987). However, the shape of P1 elements of the two species is quite different, and intermediate forms are not known. Another possibility, more likely, is that A. asymmetricus originated from A. carlsi, that has a more similar shape, by a greater development of the lateral process, but there is a short gap between the last known occurrence of $A$. carlsi and the first occurrence of $A$. asymmetricus. In fact, $A$. carlsi is reported up to the top of the transitans Zone (Corradini \& Corriga 2012), whereas A. asymmetricus enters within the lower part of the eleanorae Zone, before the first occurrence of Flajsella (Murphy \& Matti 1983).

It should be pointed out that these phylogenetical reconstructions are based on the P1 element only, because the complete apparatuses of almost all the species of Lanea and Ancyrodelloides, with the exception of A. carlsi, are still not known. Therefore the relationships between the various species, and also between the two genera, shall be precisely defined only when all the apparatuses will be reconstructed.

\section{Systematic palaeontology}

Systematic notes are restricted to necessary taxonomic remarks. For suprageneric classification the scheme proposed by Sweet (1988) is followed. Synonymy lists are limited to main and more recent captions. The entire fauna is housed in the "Walliser conodont collection" at the Geoscience Centre, Georg-August University Göttingen, under reference collection number GZG 1612. Horizon and catalogue numbers of figured specimens are given in the figure captions.

Phylum Chordata Bateson, 1886

Class Conodonta Pander, 1856

Order Ozarkodinida Dzik, 1976

Family Spathognathodontidae Hass, 1959

\section{Genus Lanea Murphy \& Valenzuela-Ríos, 1999}

Type species. - Ozarkodina eleanorae Lane \& Ormiston, 1979.

Remarks. - Genus Lanea has been established by Murphy \& Valenzuela-Ríos (1999), grouping some species of $A n$ cyrodelloides characterized by terraced, normally unornamented basal platform lobes. The authors assigned four taxa to genus Lanea: L. eleanorae (Lane \& Ormiston, 1979), L. eoeleanorae Murphy \& Valenzuela-Ríos, 1999, Lanea omoalpha Murphy \& Valenzuela-Ríos, 1999 and L. telleri (Schulze, 1968). In our opinion also A. omus (= A. omus morph $\beta$ Murphy \& Matti, 1983) is a species of Lanea.

\section{Genus Kockelella Walliser, 1957}

Type species. - Kockelella variabilis Walliser, 1957.

\section{Kockelella variabilis variabilis Walliser, 1957}

Figure 5B, C

1957 Kockelella variabilis Walliser, p. 35, pl. 1, figs 3-10.

1964 Kockelella variabilis Walliser; Walliser, p. 40, pl. 16, figs 3, 4, 6, 9, 10, 14, 15 (only).

1976 Kockelella variabilis variabilis Walliser. - Barrick \& Klapper, pp. 77-78, pl. 3, figs 12-17.

1999 Kockelella variabilis variabilis Walliser. - Serpagli \& Corradini, pp. 288-293, pl. 5, figs 1-13; pl. 6, figs 1-9; pl. 7, figs 1-7 (cum syn.).

Remarks. - P1 elements of Kockelella variabilis variabilis are distinguished from K. v. ichnusae by the characteristically branched lateral processes on both sides of the platform, and by the absence of the rim that borders the platform of $K . v$. ichnusae. In contrast with the stratigraphic distribution provided by Serpagli \& Corradini (1999) in their revision of genus Kockelella, that limited the species from the crassa to the ploeckensis zones, in Morocco $K$. v. variabilis ranges into the lower part of the siluricus Zone. A similar longer distribution have been documented also in Bohemia (Slavík et al. 2010) and in the Carnic Alps (Cellon section, our personal observation).

Figure 6. A - Wurmiella excavata (Branson \& Mehl, 1933); lateral view of P1 element GZG 1612-540C-3736-1, sample AT 7 31, transitans Zone. - B - Ozarkodina confluens (Branson \& Mehl, 1933); lateral view of P1 element GZG 1612-540C-3729-1, sample AT 7 20, Lower detortus Zone. - C - Ozarkodina confluens (Branson \& Mehl, 1933); lateral view of P1 element GZG 1612-540C-3729-2, sample AT 7 20, Lower detortus Zone. - D - Ozarkodina eosteinhornensis s.s. (Walliser, 1964); upper view of P1 element GZG 1612-477-3713-2, sample AT 3 15, Lower detortus Zone. - E - Ozarkodina eosteinhornensis s.1. (Walliser, 1964); upper view of P1 element GZG 1612-540C-3727-1, sample AT 7 15, Lower detortus Zone. - F - Zieglerodina remscheidensis (Ziegler, 1960); lateral view of P1 element GZG 1612-540C-3730-1, sample AT 7 22, Upper detortus Zone.

- G - Zieglerodina remscheidensis (Ziegler, 1960); lateral view of P2 element GZG 1612-477-3718-2, sample AT 3 18, Upper detortus Zone. - H - Zieglerodina planilingua (Murphy \& Valenzuela-Ríos, 1999); upper view of P1 element GZG 1612-540C-3795-1, sample AT 7 21b, Lower 


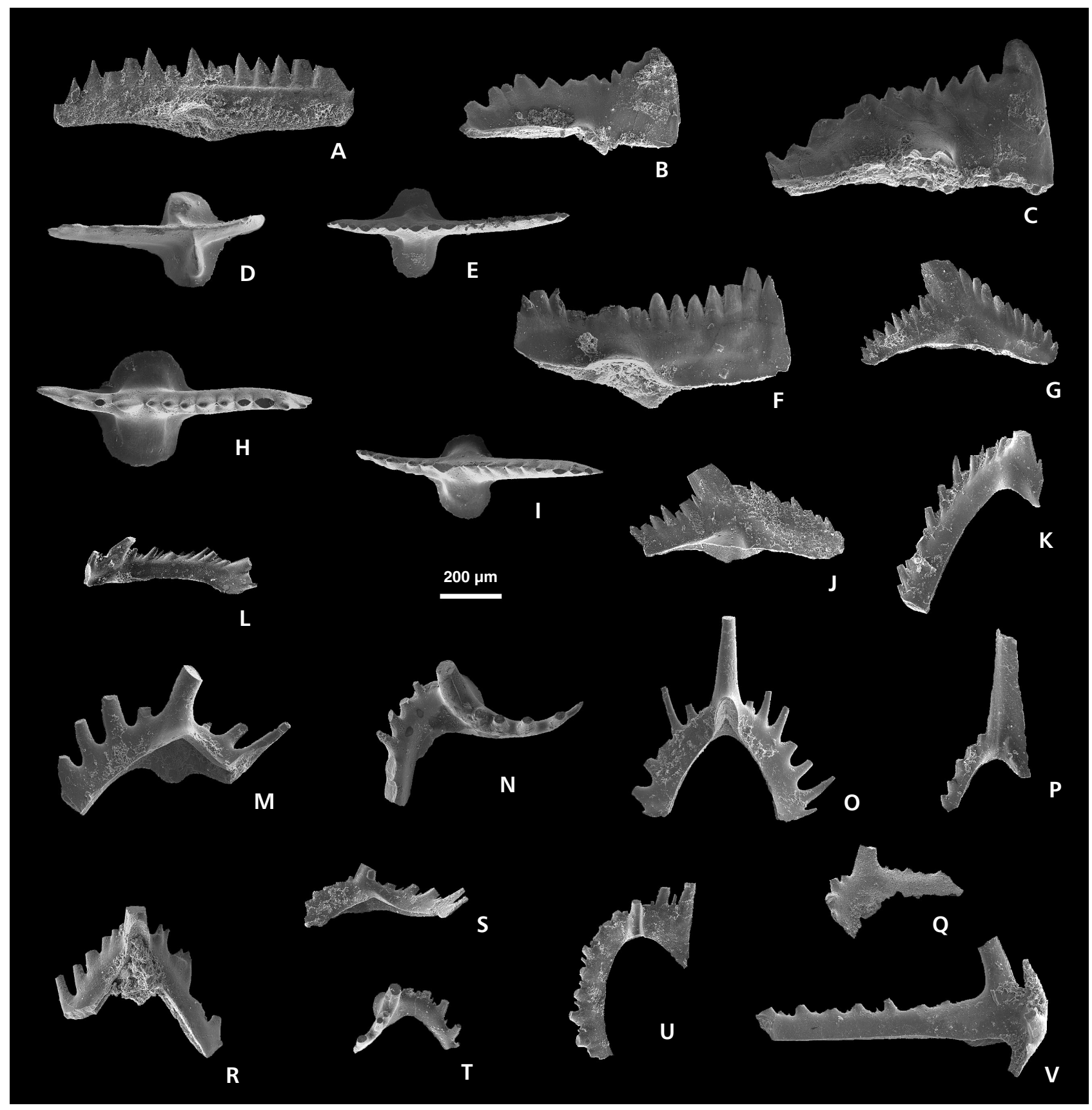

detortus Zone. • I - Zieglerodina planilingua (Murphy \& Valenzuela-Ríos, 1999); upper view of P1 element GZG 1612-540C-3720-1, sample AT 722 , Upper detortus Zone. • J - Zieglerodina planilingua (Murphy \& Valenzuela-Ríos, 1999); lateral view of P2 element GZG 1612-477-3788-1, sample AT 3 19, Upper detortus Zone. $• \mathrm{~K}$ - Zieglerodina planilingua (Murphy \& Valenzuela-Ríos, 1999); lateral view of M element GZG 1612-477-3718-3, sample AT 3 18, Upper detortus Zone. • L - Zieglerodina planilingua (Murphy \& Valenzuela-Ríos, 1999); lateral view of S1 element GZG 1612-477-3718-4, sample AT 3 18, Upper detortus Zone. • M - Oulodus elegans elegans (Walliser, 1964); lateral view of P1 element GZG 1612-540C-3727-3, sample AT 7 15, Lower detortus Zone. • N - Oulodus elegans elegans (Walliser, 1964); upper view of P2 element GZG 1612-540C-3727-4, sample AT 7 15, Lower detortus Zone. • O - Oulodus elegans elegans (Walliser, 1964); lateral view of S0 element GZG 1612-540C-3727-5, sample AT 7 15, Lower detortus Zone. • P - Oulodus elegans elegans (Walliser, 1964); lateral view of M element GZG 1612-477-3718-3, sample AT 3 18, Upper detortus Zone. • Q - Oulodus elegans elegans (Walliser, 1964); lateral view of S2 element GZG 1612-477-3708-2, sample AT 3 10b, eosteinhornensis s.1. Zone. $\bullet$ R - Oulodus sp.; lateral view of S0 element GZG 1612-477-3708-1 with anomalous development of the right process, sample AT $310 \mathrm{~b}$ eosteinhornensis s.1. Zone. $\bullet \mathrm{S}-$ Oulodus elegans detortus (Walliser, 1964); lateral view of P1 element GZG 1612-477-3710-1, sample AT 3 12, Lower detortus Zone. • T - Oulodus elegans detortus (Walliser, 1964); upper view of P2 element GZG 1612-477-3710-2, sample AT 3 12, Lower detortus Zone. • U - Oulodus elegans detortus (Walliser, 1964); lateral view of S1 element GZG 1612-477-3710-3, sample AT 3 12, Lower detortus Zone. • V - Oulodus elegans detortus (Walliser, 1964); lateral view of S2 element GZG 1612-540C-3727-6, sample AT 7 15, Lower detortus Zone. 
Stratigraphic range. - Ludlow, from within the crassa Zone (Serpagli \& Corradini 1999) to the lower part of the siluricus Zone (Slavík et al. 2010; and this paper).

Material. - 10 P1, 5 P2, 6 S0, 8 S1 and 9 S2 elements.

\section{Lanea cf. omus (Murphy \& Matti, 1983)}

Figure 5U, V

1983 Ancyrodelloides omus morph $\beta$ Murphy \& Matti, pl. 2, fig. 14, 21-29.

2012 Ancyrodelloides omus Murphy \& Matti. - Drygant \& Szaniawski, p. 858, fig.11N.

Description. - For P1 element see the description of Ancyrodelloides omus morph $\beta$ by Murphy \& Matti (1983).

P2 element strong and laterally compressed. The blade is more robust at the base of the denticles. In lateral view is arched with and angle between the processes of about $130^{\circ}$. Cusp well differentiated and oval in cross sections. Both processes bears alternate denticulation; denticles are thin and circular on the posterior process, and larger and subtriangular on the anterior one. Basal cavity wide under the cusp, where is laterally limited by a lip.

Remarks. - The P1 element fit well in the description of $L a$ nea omus morph $\beta$ by Murphy \& Matti (1983), but differs by the less expanded platform lobes: in fact, in our specimens these are narrow, resembling those of L. telleri, whereas in typical L. omus the platform lobes are wider and occupied by an evident terrace (i.e. Murphy \& Matti 1983, pl. 2, figs 24-29).

The P2 element here attributed to $L$. cf. omus is in general similar to the elements figured by Lane \& Ormiston (1979, pl. 1, fig. 47) and Murphy \& Matti (1983, pl. 4, figs 1-3) as P2 element of L. eleanorae, but differs by the less developed ledge along the processes below the denticles. However the similitude with these P2 elements of Lanea eleanorae confirms the attribution of our specimens to genus Lanea: the only species of Lanea in bed AT 7 is L. omus.

Ancyrodelloides omus was left in genus Ancyrodelloides by Murphy \& Valenzuela-Ríos (1999) when they erected the genus Lanea. However, as already suggested by Slavík (2011), the species should be moved to Lanea due to the shape of the lobes, the wide terraces and the lacking of strong ornamentation on the platform.

Stratigraphic range. - Middle Lochkovian, transitans Zone.

Material. - 1 P1 and 1 P2 elements from bed AT7 30.

\section{Genus Ozarkodina Branson \& Mehl, 1933}

Type species. - Ozarkodina confluens Branson \& Mehl, 1933.

Ozarkodina confluens (Branson \& Mehl, 1933)

Figure 6B, C

1975 Ozarkodina confluens (Branson \& Mehl). - Klapper \& Murphy, pp. 30-33, pl. 3, figs 1-23; pl. 4, figs 1-27; pl. 8, figs 11-15 (cum syn).

2009 Ozarkodina confluens (Branson \& Mehl). - Corriga \& Corradini, p. 163, fig. 4M, N.

2012 Ozarkodina confluens (Branson \& Mehl). - Corradini \& Corriga, pp. 644-645, fig. 6.

Remarks. - As evidenced by Walliser (1964, fig. 8) and by Klapper \& Murphy (1975, pp. 30-33), Ozarkodina confluens shows a wide variability of P1 element. Such variability is present also in the studied collection from Tafilalt. However, it seems that there is a very low stratigraphic value for the various morphotypes. For example, extreme forms with a strong, subtriangular shape of the blade and a large denticle at the anterior end (Fig. 6C) have been reported in literature from various levels of Ludlow and Přídolí (see discussion in Corriga \& Corradini 2009, Slavík et al. 2010).

Stratigraphic range. - Ludlow-Přídolí, from the siluricus Zone (Corradini \& Serpagli 1999) to the upper part of the Upper detortus Zone (Corradini \& Corriga 2012).

Material. - 17 P1, 2 P2 and 1 S2 elements.

\section{Genus Polygnathoides Branson \& Mehl, 1933}

Type species. - Polygnathoides siluricus Branson \& Mehl, 1933.

\section{Polygnathoides siluricus Branson \& Mehl, 1933}

Figure 5E-I

1933 Polygnathoides siluricus Branson \& Mehl, p. 50, pl. 3, figs 39-42.

1964 Polygnathoides siluricus Branson \& Mehl. - Walliser, p. 66, pl. 17, figs 1-11.

1975 Polygnathoides siluricus Branson \& Mehl. - Klapper \& Murphy, p. 56, pl. 8, figs 16-21.

1983 Polygnathoides siluricus Branson \& Mehl. - Jeppsson, fig. 1A-E.

2010 Polygnathoides siluricus Branson \& Mehl. - Slavík et al., fig. 3.

2012 Polygnathoides siluricus Branson \& Mehl. - Manda et al., fig. 4.9 . 


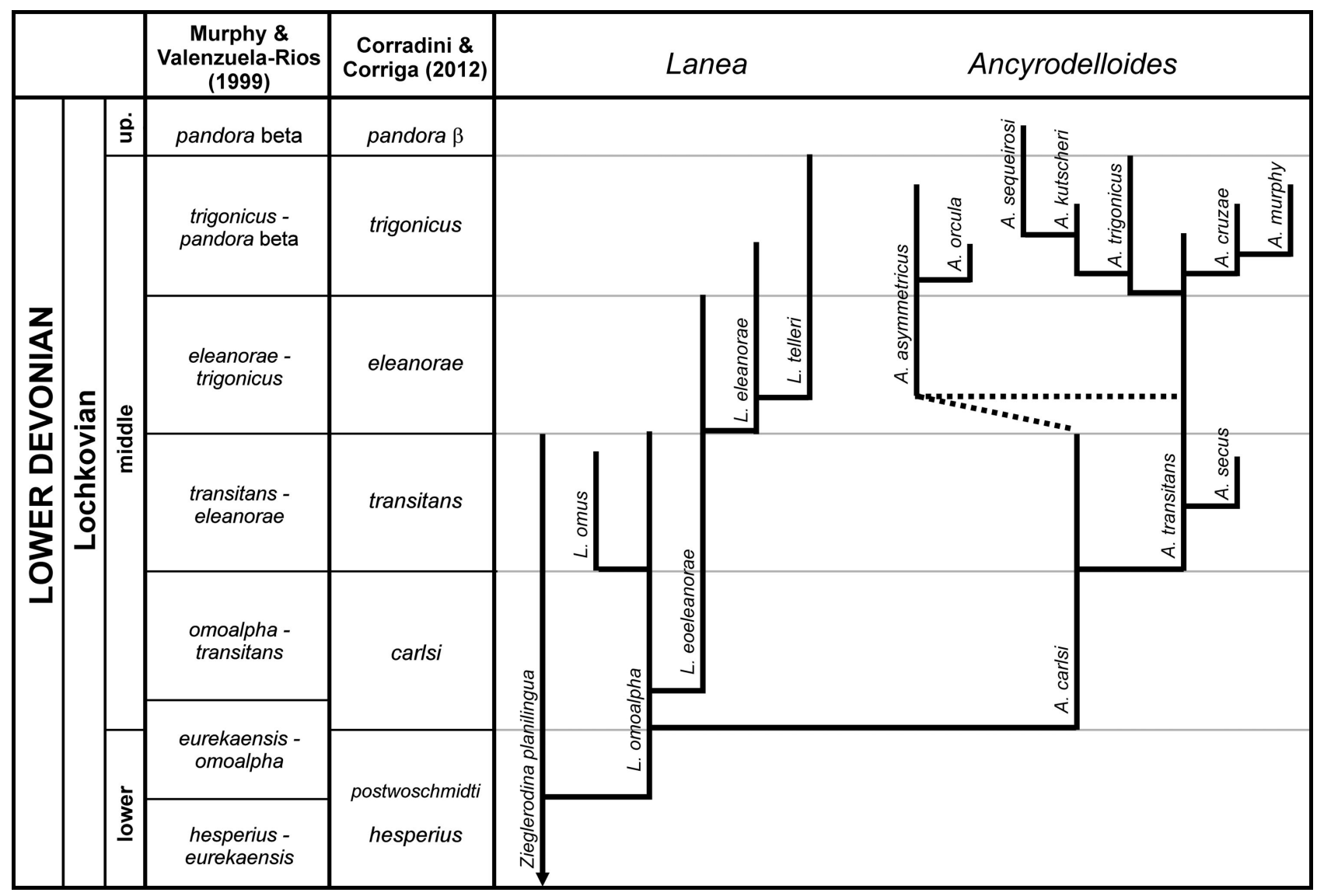

Figure 7. Reconstruction of the origin and phylogenetic relationships of genera Lanea and Ancyrodelloides.

Remarks. - The P1 element of Polygnathoides siluricus shows a wide variability in the shape of the platform. In upper view it has a more or less rhomboidal shape, with the minor axis variable in length. The upper surface can be more or less flat, or with differences of height in the central part, where a distinct crease may occur, often more pronounced on one side.

In the studied material one specimen, left in open nomenclature (Fig. 5J), has the platform limited to the anterior and central part of the element, and tapers abruptly posteriorly, where a short free blade is present. Platform margins are raised and turned upward.

Stratigraphic range. - Ludlow, siluricus Zone.

Material. - 22 P1, 12 P2, 4 M, 1 S0, 2 S1 and 4 S2 elements.

\section{Genus Zieglerodina Murphy, Valenzuela-Ríos \& Carls, 2004}

Type species. - Spathognathodus remscheidensis Ziegler, 1960.
Remarks. - Genus Zieglerodina was proposed by Murphy et al. (2004) to include the ozarkodinids of the "remscheidensis Group". Taxa of the "eosteinhornensis Group", that Murphy et al. (2004) placed in a different genus, still not defined according to the ICZN code, have a very similar morphology of all the apparatus elements. The opportunity to establish two different genera very similar each other, or to place the two groups together in the same genus should be carefully evaluated and is not the topic of this paper.

\section{Zieglerodina planilingua (Murphy \& Valenzuela-Ríos,} 1999)

Figure 6H-L

1964 Spathognathodus steinhornensis remscheidensis (Ziegler). - Walliser, pl. 20, fig. 26, 27.

1999 Ozarkodina planilingua Murphy \& Valenzuela-Ríos, p. 326, pl. 1, figs 1,9 .

2009 Ozarkodina planilingua Murphy \& Valenzuela-Ríos. - Corriga \& Corradini, fig. J, K.

2012 Zieglerodina planilingua (Murphy \& Valenzuela-Ríos). - Drygant \& Szaniawski, pp. 856-857, fig. 13A-D. 
Description. - For P1 element see Murphy \& Valenzuela-Ríos (1999).

P2 element "ozarkodiniform" with a strong cusp posteriorly reclined. The processes bears denticles discrete, with differences in size between adjacent denticles; in general denticles of anterior process are smaller than those on posterior process. Basal cavity large under the cusp, where is limited by wide lips.

Melement "neoprioniodiform" with a strong cusp, oval in cross section, with a small keel on its anterior and posterior edges. Porterior process directed downward, slightly arched, bearing thin alternate denticles. Large basal cavity under the cusp, limited by a wide lip. A small single denticle is present anteriorly of the cusp.

S1 element "plectospathodiform", laterally compressed with two asymmetrical processes. Cusp, larger than adjacent denticles, posteriorly directed. Posterior process bears denticles of different size, stronger in the distal part. Small basal cavity, surrounded by a lip under the cusp.

Remarks. - The attribution of this species to genus Zieglerodina was recently proposed by Drygant \& Szaniawski (2012). We confirm this attribution, on the basis of similitudes both in the P1 and the P2 elements with other species of Zieglerodina.

The $\mathrm{M}$ and $\mathrm{S} 1$ elements here tentatively assigned to Z. planilingua (fig $6 \mathrm{~K}, \mathrm{~L}$ ) are characterized by an evident lip that limits the basal cavity. Even if incomplete, they show characteristics of Zieglerodina, such as the alternate denticulation. These elements are different from those of $Z$. remscheidensis and of other ozarkodinids present in these samples, and therefore their attribution to Z. planilingua is very likely.

Having Z. planilingua a wide geographic distribution in Europe, North America and Australia, the first occurrence of the species can be a good stratigraphic marker in the middle Přídolí.

Stratigraphic range. - From the Lower detortus Zone (eosteinhornensis s.s. horizon) to the transitans Zone (Corradini \& Corriga, 2012).

Material. - 117 P1, 9 P2, 2 M and 3 S1 elements.

\section{Conclusions}

The main results of this study on Silurian and Devonian conodonts from three section in the Tafilalt can be summarized as follows. In terms of stratigraphy several conodont zones from Ludlow to Lochkovian have been documented, allowing stating that:

- the age of the "Orthoceras limestones" in Tafilalt is confirmed as middle Ludlow (ploeckensis and siluricus zones);
- the "Scyphocrinites limestones" spans the Silurian/ Devonian boundary, from the Přídolí to lower Lochkovian (eosteinhornensis s.1.-hesperius zones);

- a couple of beds of Orthoceras Limestone occur also within the middle Lochkovian (transitans Zone), only in the Atrous 7 section.

In terms of conodont taxonomy

- some elements possibly belonging to the apparatus of Zieglerodina planilingua (Murhpy \& Valenzuela-Ríos, 1999) are described;

- the species Ancyrodelloides omus Murphy \& Matti, 1983 has been moved to genus Lanea, and a P2 element has been tentatively assigned to the species;

- the phylogenetic relationships between genera Lanea and Ancyrodelloides are suggested.

\section{Acknowledgements}

This paper is dedicated to our good friend Mena Schemm-Gregory. This study would not have been possible without the detailed work of Otto H. Walliser, who examined the sections and collected the conodont samples. Helga Groos-Uffenorde (Göttingen) is deeply acknowledged for help in the preparation of the manuscript by examination of maps, field notes and manuscripts in the asset of O.H. Walliser. Reimund Haude (Göttingen) provided useful information on the sections and the occurrence of crinoids. Gabriele Cruciani (Cagliari) took SEM photos of figured specimens. Ladislav Slavík (Prague) and Thomas Suttner (Graz) reviewed the manuscript providing useful comments. This research was partly supported by grants RAS (resp. C. Corradini). This paper is a contribution to IGCP projects No. 591 (The Early to Middle Paleozoic revolution) and 596 (Climate change and biodiversity patterns in the Mid-Paleozoic).

\section{References}

BARRICK, J.E. \& KLAPPER, G. 1976. Multielement (late Llandoverian-Wenlockian) conodonts ot the Clarita Formation, Arbuckle Mountains, Oklahoma, and phylogeny of Kockelella. Geologica et Palaeontologica 10, 59-100.

BARRICK, J.E. \& KLAPPER, G. 1992. Late Silurian-Early Devonian conodonts from the Hunton Group (Upper Henryhouse, Haragan, and Bois d'Arc Formations), South-Central Oklahoma. Oklahoma Geological Survey Bulletin 145, 19-65.

Barrick, J.E., Meyer, B.D. \& Ruppel, S.C. 2005. The Silurian-Devonian boundary and the Klonk Event in the Frame Formation, subsurface West Texas, 105-122. In BARRICK, J.E. \& LANE, H.R. (eds) A standing ovation: Papers on honour of Gilbert Klapper. Bulletin of American Paleontology 369.

BAteson, W. 1886. Anchestry of the Chordata. Quarterly Journal of Microscopical Sciences 26, 535-571.

Becker, R.T., Aboussalam, Z.S., Baider, L., El Hassani, A. \& STichling, S. 2013. The Lower and Middle Devonian at El Khraouia (Southern Tafilalt), 31-40. In BECKER, R.T., EL HASSANI, A. \& TAHIRI, A. (eds) International Field Symposium 
"The Devonian and Carboniferous of Northern Gondwana" Morocco 2013. Documents de l'Institut Scientifique, Rabat 27.

Belka, Z., Klug, K., Kaufmann, B., Korn, D., Döring, S., Feist, R. \& WeNDT, J. 1999. Devonian condont and ammoniod succession of eastern Tafilalt (Ouidane Chebbi section), Anti-Atlas, Morocco. Acta Geologica Polonica 49, 1-23.

Bischoff, G. \& Sannemann, D. 1958. Unterdevonische Conodonten aus dem Frankenwald. Notizblatt des Hessischen Landesamtes für Bodenforschung 86, 87-110.

Boersma, K.T. 1973. Description of certain Lower Devonian platform conodonts of the Spanish Central Pyrenees. Leidse Geologische Mededelingen 49, 285-301.

Branson, E.B. \& Meht, M.G. 1933. Conodonts from the Bainbridge Formation (Silurian) of Missouri. University of Missouri Studies 8, 39-52.

Carls, P. \& Gandl, J. 1969. Stratigraphie und Conodonten des Unter-Devons der Östlichen Iberischen Ketten (NE Spanien). Neues Jahrbuch für Geologie und Paläontologie, Abhandlungen 132, 155-218.

Corradini, C. \& Corriga, M.G. 2010. Silurian and lowermost Devonian conodonts from the Passo Volaia area (Carnic Alps, Italy). Bollettino della Società Paleontologica Italiana 49(3), 237-253.

Corradini, C. \& Corriga, M.G. 2012. A Přídolí-Lochkovian conodont zonation in Sardinia and the Carnic Alps: implications for a global zonation scheme. Bulletin of Geosciences 87(4), 635-650. DOI 10.3140/bull.geosci.1340

Corradini, C., Ferretti, A., Corriga, M.G. \& Serpagli, E. 2009a. The reference section of the Sardinian Ockerkalk: the Silius Section. Rendiconti della Società Paleontologica Italiana 3(2), 209-216.

Corradini, C., Ferretti, A., Corriga, M.G. \& Serpagli, E. 2009b. Loboliths (crinoids) and conodont biostratigraphy of the Genna Ciuerciu Section (SE Sardinia). Rendiconti della Società Paleontologica Italiana 3(2), 217-224.

Corradini, C., Olivieri, R. \& Serpagli, E. 1996. Possible relationship between anomalous conodonts and Silurian oceanic episodes. Neues Jahrbuch für Geologie und Paläontologie, Monatshefte H 12, 737-746.

Corradini, C. \& Serpagli, E. 1999. A Silurian conodont zonation from late Llandovery to end Prídoli in Sardinia. Bollettino della Società Paleontologica Italiana 38(2-3), 255-273.

Corriga, M.G. 2011. Biostratigrafia a conodonti attorno al limite Siluriano-Devoniano in alcune aree del Nord Gondwana. 174 pp. Ph.D. thesis, Università di Cagliari, Italy.

Corriga, M.G. \& Corradini, C. 2009. Upper Silurian and Lower Devonian conodonts from the Monte Cocco II section (Carnic Alps, Italy). Bulletin of Geosciences 84(1), 155-168. DOI 10.3140/bull.geosci.1112

Corriga, M.G., Corradini, C. \& Ferretti, A. 2009. Silurian conodonts from Sardinia: an overview. Rendiconti della Società Paleontologica Italiana 3(1), 95-107.

Corriga, M.G., Corradini, C., Haude, R. \& Walliser, O.H. 2013. Upper Silurian and Lower Devonian conodonts and crinoids from the scyphocrinoid beds of southeastern Morocco, 71-72. In Lindskog, A. \& MeHLQvist, K. (eds) Proceedings of the $3^{\text {rd }}$ IGCP 591 Annual Meeting, Lund, Sweden, 9-19 June 2013.
Corriga, M.G., Corradini, C., Haude, R. \& Walliser, O.H. 2014. Conodonts and crinoids stratigraphy of the Upper Silurian and Lower Devonian scyphocrinoid beds of Tafilalt, southeastern Morocco. GFF, 1-5.

DOI 10.1080/11035897.2013.862849

Corriga, M.G., Corradini, C., Pondrelli, M., \& Simonetto, L. 2012. Lochkovian (Lower Devonian) conodonts from the Rio Malinfier section (Carnic Alps, Italy). Gortania Geologia, Paleontologia, Paletnologia 33, 31-38.

Corriga, M.G., Suttner, T.J., Kido, E., Corradini, C., PondRelli, M. \& Simonetto, L. 2011. The La Valute limestone-Findenig limestone transition in the La Valute Section (Lower Devonian, Carnic Alps, Italy). Gortania Geologia, Paleontologia, Paletnologia 32, 5-12.

Cramer, B.D., Brett, C.E., Melchin, M.J., Männik, P., Kleffner, M.A., McLaughlin, P.I., Loydell, D.K., Munnecke, A., Jeppsson, L., Corradini, C., Brunton, F.R. \& Saltzman, M.R. 2011. Revised correlation of Silurian Provincial series of North America with global and regional chronostratigraphic units and $\delta^{13} \mathrm{C}_{\text {carb }}$ chemostratigraphy. Lethaia 44, 185-202. DOI 10.1111/j.1502-3931.2010.00234.x

Drygant, D.M. 1974. Prostye konodonty silura i nizov devona Volyno-Podol'ya [Simple conodonts from the Silurian and lowermost Devonian of Volyno-Podolia]. Paleontologicheskii sbornik 10, 64-70. [in Russian]

Drygant, D. \& SzANiAwski, H. 2012. Lochkovian conodonts from Podolia, Ukraine and their stratigraphic significance. Acta Palaeontologica Polonica 57(4), 833-861.

DzIK, J. 1976. Remarks on the evolution of Ordovician conodonts. Acta Palaeontologica Polonica 21, 395-455.

Hass, W.H. 1959. Conodonts from the Chappel Limestone of Texas. U.S. Geological Survey Professional Paper 294, 365-399.

Haude, R. \& Walliser, O.H., 1998. Conodont-based Upper Silurian-Lower Devonian range of scyphocrinoids in SE Morocco, 94-96. In Gutiérrez-Marco, J.C. \& RÁbano, I. (eds) Proceedings of the $6^{\text {th }}$ International Graptolite Conference and 1998 Field Meeting IUGS Subcommission on Silurian Stratigraphy. Temas Geológico-Mineros ITGE 23.

Helfrich, C.T. 1980. Silurian conodonts from Wills Mountain Anticline, Virginia, West Virginia, and Maryland. Geological Society of America Special Paper 161, 1-82 + appendix 86 pp.

Hollard, H. 1977. Le domaine de l'Anti-Atlas au Maroc, 168-194. In Martinsson, A. (ed.) The Silurian-Devonian boundary. IUGS Series A 5.

JEPPSSON, L. 1983. Silurian conodont faunas from Gotland. Fossils and Strata 15, 121-144.

KLAPPER, G. \& Murphy, M.A. 1975. Silurian-Lower Devonian conodont sequence in the Roberts Mountains Formation of central Nevada. University of California Publications in Geological Sciences 111, 1-62.

KRÖGER, B. 2008. Nautiloids before and during the origin of ammonoids in a Siluro-Devonian section of the Tafilalt, AntiAtlas, Morocco. Special Papers in Palaeontology 79, 1-110.

LANE, H.R. \& ORMiston, A.E. 1979. Siluro-Devonian biostratigraphy of the Salmontrout River area, east-central Alaska. Geologica et Palaeontologica 13, 39-96.

Manda, S̆., S̆ToRCh, P., Slavík, L., FrÝdA, J., KŘíž, J. \& TASÁRYOVÁ, Z. 2012. Graptolite, conodont and sedimentary record 
through the late Ludlow Kozlowskii Event (Silurian) in shale dominated succession of Bohemia. Geological Magazine 149, 507-531. DOI 10.1017/S0016756811000847

Mavrinskaya, T. \& Slavík, L. 2013. Correlation of Early Devonian (Lochkovian-early Pragian) conodont faunas of the South Urals (Russia). Bulletin of Geosciences 88(2), 283-296. DOI 10.3140/bull.geosci.1404

Mashkova, T.V. 1968. The conodonts of the genus Icriodus Branson \& Mehl, 1938 from the Borshchiv and Chorkiv Horizons of Podolia. Doklady AN SSSR 182, 941-944. [in Russian]

Murphy, M.A. \& Cebecioglu, M.K. 1987. Morphometric study of the genus Ancyrodelloides (Lower Devonian, conodonts) central Nevada. Journal of Paleontology 61, 683-594.

Murphy, M.A. \& MatTI, J.C. 1983. Lower Devonian conodonts (hesperius-kindlei zones), central Nevada. University of California Publications in Geological Sciences 123, 1-83.

Murphy, M.A. \& ValenZuela-Ríos, J.I. 1999. Lanea new genus, lineage of Early Devonian conodonts. Bollettino della Società Paleontologica Italiana 37, 321-334.

Murphy, M.A., Valenzuela-Ríos, J.I. \& Carls, P. 2004. On Classification of Pridoli (Silurian)-Lochkovian (Devonian) Spathognathodontidae (Conodonts). University of California, Riverside Campus Museum Contribution 6, 1-25.

PANDER, C.H. 1856. Monographie der fossilen Fische des Silurischen System der Russisch-Baltischen Gouvernments. 83 pp. Petersburg.

Rytina, M.-K., Becker, R.T., Aboussalam, Z.S., Hartenfelds, S., Helling, S., Stichling, S. \& Ward, D. 2013. The allochtonous Silurian-Devonian in olistostromes at "the Southern Variscan front" (Tinerhir region, SE Morocco) - Preliminary data, 11-21. In BeCKer, R.T., El Hassani, A. \& TAhiRI, A. (eds) International Field Symposium "The Devonian and Carboniferous of Nortern Gondwana" - Morocco 2013. Documents de l'Institut Scientifique, Rabat 27.

Schulze, R. 1968. Die Conodonten aus dem Paläozoikum der mittleren Karawanken (Seeberggebiet). Neues Jahrbuch für Geologie und Paläontologie, Abhandlungen 130(2), 133-245.

Serpagli, E. \& Corradini, C. 1998. New taxa of Kockelella (Conodonta) from Late Wenlock-Ludlow (Silurian) of Sardinia, 79-83. In Serpagli, E. (ed.) Sardinia Field-trip Guidebook, ECOS VII, Giornale di Geologia 60, Spec. Issue.

SERPagli, E. \& Corradini, C. 1999. Taxonomy and evolution of Kockelella (Conodonta) from Silurian of Sardinia. Bollettino della Società Paleontologica Italiana 38(2-3), 275-298.

SLAVÍK, L. 2011. Lanea carlsi conodont apparatus reconstruction and its significance for subdivision of the Lochkovian. Acta
Palaeontologica Polonica 56(2), 313-327.

DOI 10.4202/app.2009.0046

Slavík, L., Carls, P, Hladil, J. \& Koptíková, L. 2012. Subdivision of the Lochkovian Stage based on conodont faunas from the stratotype area (Prague Synform, Czech Republic). Geological Journal 47, 616-631. DOI 10.1002/gj.2420

SlavíK, L., KŘiž, J. \& Carls, P. 2010. Reflection of the midLudfordian Lau Event in conodont faunas of Bohemia. Bulletin of Geosciences 85(3), 395-414.

DOI 10.3140/bull.geosci.1204

SLAVÍK, L., ŠTORCh, P., MANDA, Š. \& FrÝdA, J. 2013 (in press). Integrated stratigraphy of the Ludfordian in the Prague Synform. GFF. DOI 10.1080/11035897.2013.851733

SWEET, W.C. 1988. The Conodonta: morphology, taxonomy, paleoecology, and evolutionary history of a long-extinct animal phylum. Oxford Monographs on Geology and Geophysics 10. 212 pp. Oxford University Press, New York.

VAlenZuela-Ríos, J.I. 1994. Conodontos del Lochkoviense y Praguiense (Devónico Inferior) del Pirineo Central Español. Memorias del Museo Paleontologico de la Universidad de Zaragoza 5, 1-142.

ValenZuela-Ríos, J.I. 1999. Ancyrodelloides sequeirosi, un nuevo conodonto del Lochkoviense medio (Dévonico Inferior) de los Pirineos orientales españoles, 247-253. In GÁmEZ VinTANED, J.A. \& LiÑÁn, E. (eds) VI Jornadas Aragonesas de Paleontología. 25 anos de Paleontología Aragonesa. Homenaje al profesor Leandro Sequeiros.

WALliser, O.H. 1957. Conodonten aus dem oberen Gotlandium Deutschlands und der Karnischen Alpen. Notizblatt des Hessischen Landesamtes für Bodenforschung zu Wiesbaden 85, $28-52$.

WalLiser, O.H. 1964. Conodonten des Silurs. Abhandlungen des Hessischen Landesamtes für Bodenforschung zu Wiesbaden 41, 1-106.

Wilson, G. 1989. Documentation of conodont assemblages across the Lochkovian-Pragian (Early Devonian) Boundary at Wellington, Central New South Wales, Australia, 117-171. In ZIEGLER, W. (ed.) $I^{\text {st }}$ International Senckenberg Conference and $5^{\text {th }}$ European Conodont Symposium (ECOS V) - Contribution III. Courier Forschungsinstitut Senckenberg 117.

ZIEGLER, W. 1960. Conodonten aus dem Rheinischen Unterdevon (Gedinnium) des Remscheider Sattels (Rheinisches Schiefergebirge). Palaontologische Zeitschrift 34(2), 169-201. DOI 10.1007/BF02987050

ZiegleR, W. 1991. Catalogue of conodonts. Volume V, 17. 445 pp. Schweizerbart'sche Verlagsbuchhandlung, Stuttgart. 